\title{
Discovery of LYS006, a Potent and Highly Selective Inhibitor of Leukotriene $\mathrm{A}_{4}$ Hydrolase
}

Christian Markert, ${ }^{*}, a$ Gebhard Thoma, ${ }^{a}$ Honnappa Srinivas, ${ }^{b}$ Birgit Bollbuck, ${ }^{a}$ Rainer M. Lüönd, ${ }^{a}$ Wolfgang Miltz, ${ }^{a}$ Rudolf Wälchli, ${ }^{a}$ Romain Wolf, ${ }^{a}$ Jürgen Hinrichs, ${ }^{a}$ Christian Bergsdorf, ${ }^{b}$ Kamal Azzaoui, ${ }^{b}$ Carlos A. Penno, ${ }^{c}$ Kai Klein, ${ }^{d}$ Nathalie Wack, ${ }^{e}$ Petra Jäger, ${ }^{e}$ Franziska Hasler, ${ }^{e}$ Christian Beerli, ${ }^{e}$ Pius Loetscher, ${ }^{e}$ Janet Dawson, ${ }^{e}$ Grazyna Wieczorek, ${ }^{e}$ Shin Numao, ${ }^{b}$ Amanda Littlewood-Evans, ${ }^{e}$ and Till A. Röhn

${ }^{\mathrm{a}}$ Global Discovery Chemistry, ${ }^{\mathrm{b}}$ Chemical Biology and Therapeutics, ${ }^{\mathrm{c}}$ Analytical Sciences \& Imaging, ${ }^{\mathrm{d} P K}$ Sciences, e Autoimmunity, Transplantation and Inflammation, Novartis Institutes for Biomedical Research, 4002 Basel, Switzerland

\section{Supporting Information}

\section{Table of Contents:}

Chemistry

UPLC traces of compounds 1-23

Table S1. Smiles and data for compounds 1-30

Table S2. Inhibitory activity of LYS006 against other Zn-containing enzymes

Structural Biology

References
$\mathrm{S} 2-\mathrm{S} 24$

S25

S34

S35

S36 - S39

S40 


\section{CHEMISTRY}

All chemicals were used directly as received from commercial suppliers. Reference compounds

DG-051, JNJ-40929837 and Acebilustat were synthesized according to published procedures. ${ }^{1-3}$ NMR spectra were recorded on Bruker 400 or $600 \mathrm{MHz}$ spectrometers. Chemical shifts are expressed in $\delta \mathrm{ppm}$ referenced to the residual solvent peak (DMSO- $\mathrm{d}_{6}, \delta=2.50 \mathrm{ppm}$; $\mathrm{CDCl}_{3}$, $\delta=7.27 \mathrm{ppm}$; $\mathrm{CD}_{3} \mathrm{OD}, \delta=3.31 \mathrm{ppm}$ ). Abbreviations used in describing peak signals are $\mathrm{br}=$ broad signal, $\mathrm{s}=$ singlet, $\mathrm{d}=$ doublet, $\mathrm{dd}=$ doublet of doublets, $\mathrm{t}=$ triplet, $\mathrm{q}=$ quartet, $\mathrm{m}=$ multiplet. All final compounds were purified to $\geq 95 \%$ by reverse phase high performance liquid chromatography (HPLC), supercritical fluid chromatography (SFC), normal or reverse phase column chromatography. Purities were assessed by reverse phase UPLC with a gradient of 5-60\% isopropanol in water (with either acid or base modifier) and monitored by UV absorption at 210-450 nm. Optical purity was determined by HPLC (Agilent 1100 COOK) on chiral stationary phase. Low-resolution mass spectra were recorded on a liquid chromatography-mass spectrometer in electrospray positive (ESI+) and negative (ESI-) modes. HR-MS analyses were performed by using electrospray ionization in positive ion mode after separation by liquid chromatography (UltiMate 3000 from Dionex). The elemental compositions were derived from the mass spectra acquired at a high resolution of about 70000 on a QExactive Plus mass spectrometer (Thermo Scientific). The high mass accuracy below 1 ppm was obtained by using a lock mass. 


\section{ABBREVIATIONS}

aq: aqueous

BOC: tert-butoxycarbonyl

DCM: dichloromethane

DIAD: diisopropyl azodicarboxylate

DIPEA: diisopropylethylamine

DMF: dimethylformamide

DMSO: dimethylsulfoxide

ESI: electrospray ionization

h: hour(s)

HATU: 1-[bis(dimethylamino)methylene]-1H-1,2,3-triazolo[4,5-b]pyridinium 3-oxid hexafluorophosphate

M: molar

min: minute(s)

MMP: matrix metalloproteinase

$\mathrm{RM}$ : reaction mixture

rt: room temperature

sat. : saturated

SFC: supercritical fluid chromatography

TACE: tumor necrosis factor- $\alpha$ converting enzyme

TBAF: tetrabutylammonium fluoride

TBME: tert-butyl methylether

TFA: trifluoroacetic acid

THF: tetrahydrofuran

TMS: trimethylsilyl 


\section{SYNTHESES}

The syntheses of amines $\mathbf{2 - 4}$ is outlined in Scheme S1. Tetrazoles S-2 and S-4 were prepared using a tin catalyzed cycloaddition of TMS-azide as described for 27. Alkylation of the tetrazoles occurred with good regioselectivity affording the protected amines, which were isolated (S-1 and S-3) or directly deprotected (S-5) affording amines 2-4.

Scheme S1: Preparation of amines 2-4. ${ }^{a}$

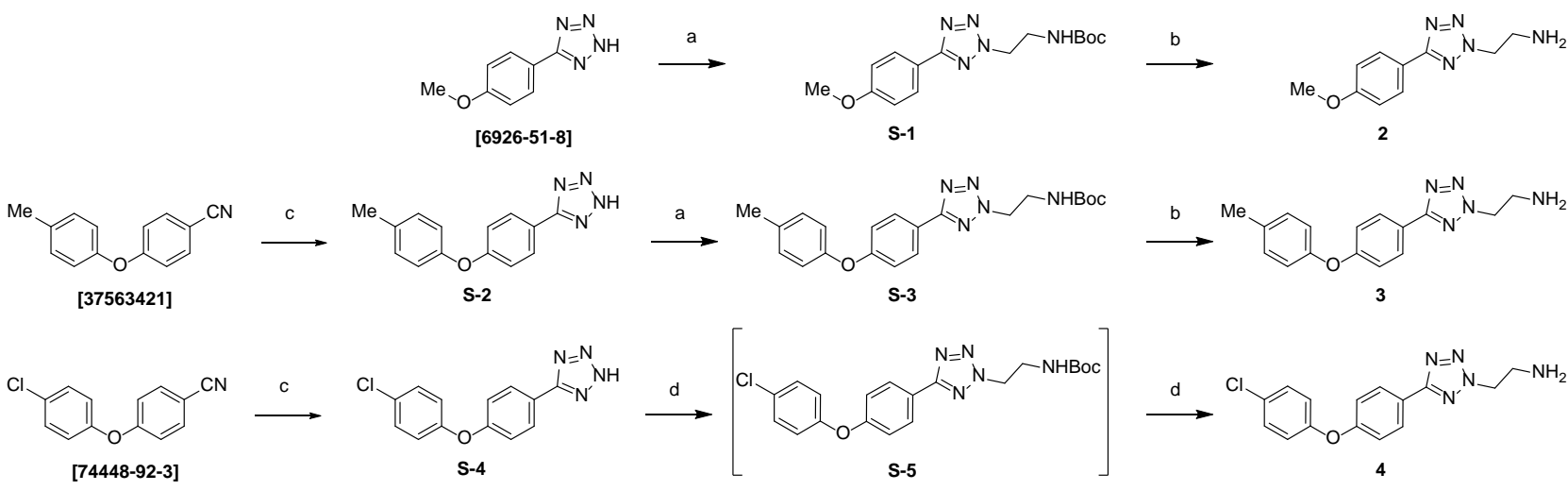

${ }^{a}$ Reagents and conditions: (a) tert-butyl 1,2,3-oxathiazolidine-3-carboxylate 2,2-dioxide, DIPEA, DMF, rt, 2 h, S-1:71\%, S-3: 63\%; (b) TFA, DCM, rt, 1 h, 2: 91\%, 3: 63\%; (c) $\mathrm{Bu}_{2} \mathrm{SnO}, \mathrm{TMSN}_{3}$, toluene, $90^{\circ} \mathrm{C}, 18$ h, S-2: 45\%, S-4: 86\%; (d) ROH, $\mathrm{PPh}_{3}$, di-tert-butyl azodicarboxylate, THF, rt, 3 h; then TFA, DCM, rt, 2 h, 74\%, two steps.

Scheme S2 summarizes the syntheses of derivatives 5-13. With two exceptions, all syntheses start with the alkylation of tetrazole S-4 installing an aliphatic chain that carries a protected head-group, followed by a final deprotection step. Alkyl halides (9), sulfamidates [S-6, (S)-S-11] and alcohols under Mitsunobu conditions [S-7, S-8, S-9, (R)-S-11] gave similarly good conversions and regioselectivities. Low yields were observed for reagents and products that are prone to undergo E1 $1_{c b}$ elimination under basic conditions $(\mathbf{1 0}, \mathbf{1 1})$. Acetamide 6 was obtained in good yield under standard conditions. The alkylation of $\mathbf{4}$ leading to S-10 gave dominant mono-alkylation, and the mediocre yield likely only originates from losses during purification. 
Scheme S2: Preparation of derivatives 5-13. ${ }^{a}$
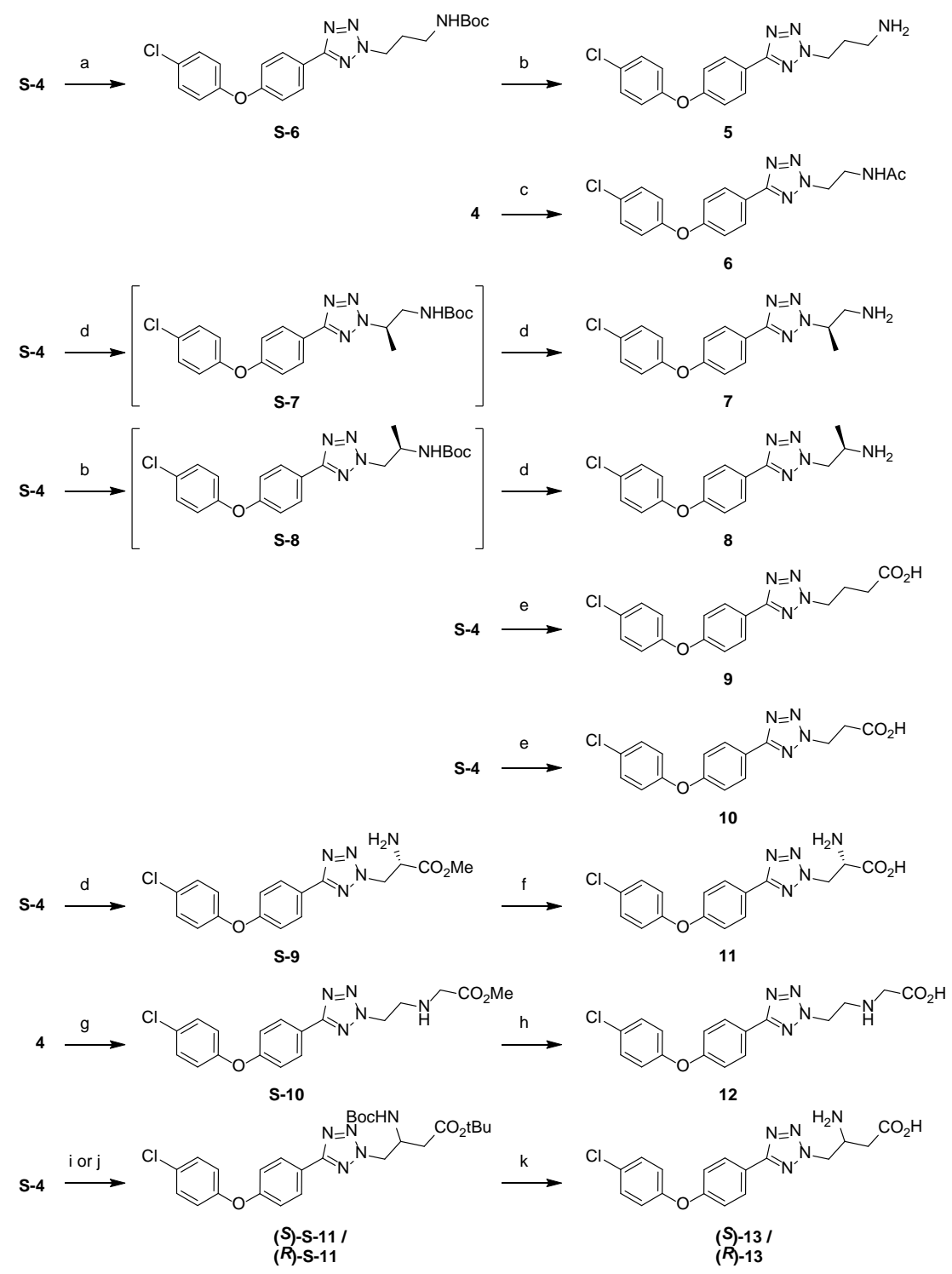

${ }^{a}$ Reagents and conditions: (a) tert-butyl 1,2,3-oxathiazinane-3-carboxylate 2,2-dioxide, DIPEA, DMF, rt, 2 h, 81\%; (b) TFA, DCM, rt, 1 h, 87\%; (c) Ac2 O, NEt3, DCM, rt, 18 h, 79\%; (d) ROH, $\mathrm{PPh}_{3}$, di-tert-butyl azodicarboxylate, THF, rt, 2 h; then TFA, DCM, rt, 1 h, 7: 67\%, 8: 78\%, S-9: 63\%, two steps; (e) $\mathrm{Br}\left(\mathrm{CH}_{2}\right)_{3} \mathrm{CO}_{2} \mathrm{Et}$ or $\mathrm{Br}\left(\mathrm{CH}_{2}\right)_{2} \mathrm{CO}_{2} \mathrm{Me}, \mathrm{K}_{2} \mathrm{CO}_{3}$, DMF, rt, $18 \mathrm{~h}$; then $\mathrm{LiOH}$ rt, $2 \mathrm{~h}$, 9: 52\%, 10: 7\%; (f) $\mathrm{LiOH}, \mathrm{MeOH}$, rt, 90 min, 7\%; (g) $\mathrm{BrCH}_{2} \mathrm{CO}_{2} \mathrm{Me}, \mathrm{Cs}_{2} \mathrm{CO}_{3}, \mathrm{MeCN}, \mathrm{rt}, 3$ h, 30\%; (h) LiOH, THF, 60 C, 30 min, 46\%. (i) (S)-S-11: (S)-29, DMF, DIPEA, rt, 17 h, 97\%; (j) (R)-S-11: (R)-28, $\mathrm{PPh}_{3}$, DIAD, THF, rt, 90 min, yield n.d.; (k) $\mathrm{HCl}$ in dioxane, $45^{\circ} \mathrm{C}$ or $80^{\circ} \mathrm{C}, 3 \mathrm{~h}$, (S)-13: 76\%, (R)-13: 55\%, two steps. 
Scheme S3: Preparation of zwitterions 14-22. ${ }^{a}$

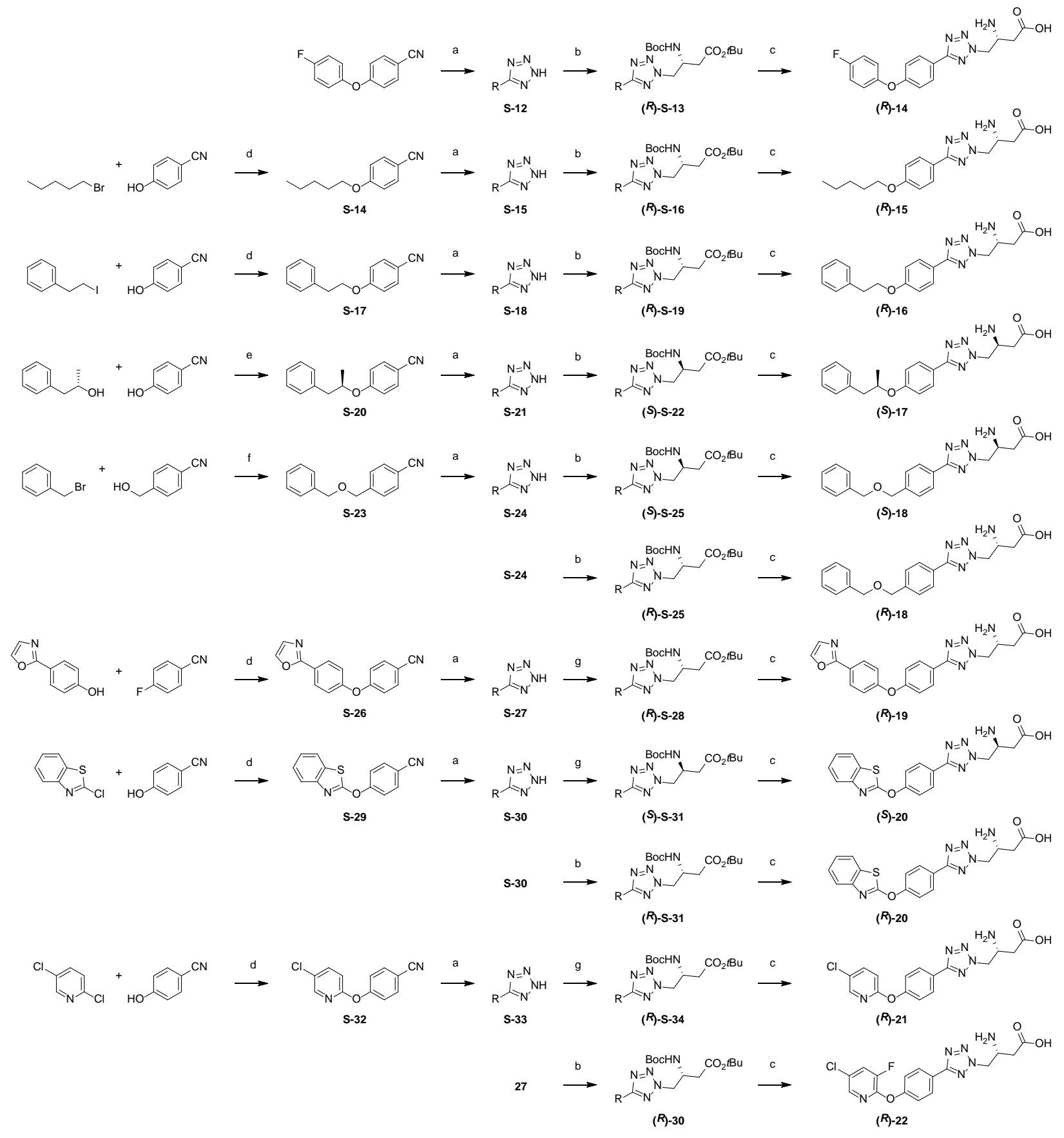

${ }^{a}$ Reagents and conditions: (a) $\mathrm{Bu}_{2} \mathrm{SnO}(10 \%)$, $\mathrm{TMSN}_{3}$, toluene, $90^{\circ} \mathrm{C}, 18 \mathrm{~h}, \mathbf{S - 1 2 :} 79 \%$, S-15: $72 \%$, S-18: 47\%, S-21: 66\%, S-24: 80\%, S-27: 78\%, S-30: 87\%, S-33: 77\%; (b) (R)-29 or (S)-29, DMF, DIPEA, RT, 15 h, (R)-S-13: 79\%, (R)-S-16: 82\%, (R)-S-19: 88\%, (S)-S-22: 99\%, (S)-S-25: 94\%, (R)-S-25: 78\%, (R)-S-31: 66\%, (R)-30: 88\%; (c) HCl in dioxane, 45ㄷ, 2 h, (R)-14: 73\%, (R)-15: 87\%, (R)-16: 83\%, (S)-17: 71\%, (S)-18: 78\%, (R)-18: 89\%, (R)-19: 71\%, (S)-20: 65\%, (R)-20: 
63\%, (R)-21: 80\%, (R)-22: 92\%; (d) $\mathrm{K}_{2} \mathrm{CO}_{3}$, DMF, S-14 (rt, 72 h): 59\%, S-17 (65ㄷ, 40 h): 33\%, S-26 (100 ${ }^{\circ} \mathrm{C}, 16$ h): 86\%, S-29 (120 C, 18 h): 49\%, S-32 (140 C, 48 h): 39\%; (e) $\mathrm{PPh}_{3}$, DIAD, 2MeTHF, rt, 3 h, 51\%; (f) KOtBu, DMF, 0-5ㄷ, 84\%; (g) (R)-28, $\mathrm{PPh}_{3}, \mathrm{DIAD}, \mathrm{THF}, \mathrm{rt}, 2$ h, (R)-S-28: 95\%, (S)-S-31: 62\%, (R)-S-34: 59\%.

Scheme S3 summarizes the syntheses of compounds 14-22. Each derivative required typically four synthetic steps with diversity introduced at the beginning. The ether bond was readily formed using alkylations (S-14, S-17), a Mitsunobu reaction under inversion of stereoconfiguration (S-20), or $\mathrm{S}_{\mathrm{N}} \mathrm{Ar}$ conditions (S-23, S-26, S-29, S-32). Optimized conditions a-c were identical for all derivatives delivering the zwitterionic final products as $\mathrm{HCl}$ salts. This typically allowed for isolation by filtration and final purification by washing with aprotic solvents.

Scheme S4 shows an alternative build-up which was used in the synthesis of (R)-23. The same transformations as described before starting from a TBS-protected phenol afforded phenol $(\boldsymbol{R})$-S-37. This intermediate could then be used in an ether formation introducing diversity at a later stage.

Scheme S4: Preparation of $(R)-23 .^{a}$
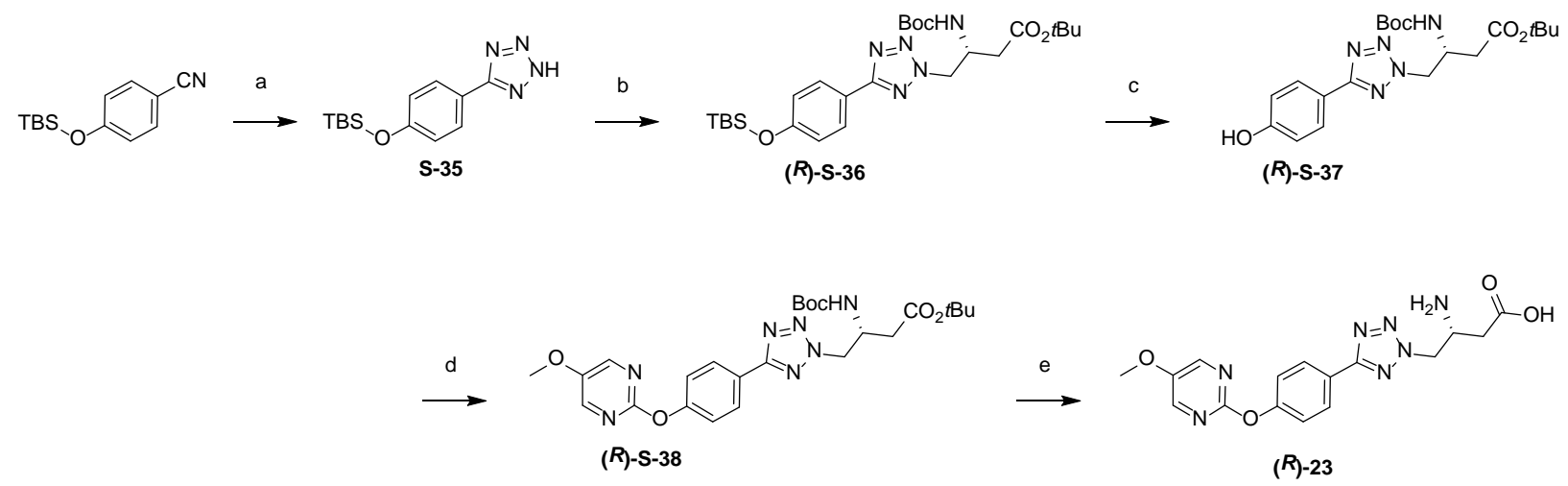

${ }^{a}$ Reagents and conditions: (a) $\mathrm{Bu}_{2} \mathrm{SnO}(10 \%)$, $\mathrm{TMSN}_{3}$, toluene, $100^{\circ} \mathrm{C}, 18 \mathrm{~h}, 92 \%$; (b) (R)-28, $\mathrm{PPh}_{3}$, DIAD, THF, rt, 6 h, 36\%; (c) TBAF, THF, 0 C, 2 h, 74\%; (d) 2-chloro-5-methoxypyrimidine, $\mathrm{Cs}_{2} \mathrm{CO}_{3}, \mathrm{DMF}, 90^{\circ} \mathrm{C}, 3 \mathrm{~h}, 72 \%$; (e) $\mathrm{HCl}$ in dioxane, $45^{\circ} \mathrm{C}, 3 \mathrm{~h}, 61 \%$. 


\section{Experimental Details}

tert-Butyl (2-(5-(4-methoxyphenyl)-2H-tetrazol-2-yl)ethyl)carbamate (S-1)

A solution of 5-(4-methoxyphenyl)-2H-tetrazole (300 mg, $1.70 \mathrm{mmol}$ ) and tert-butyl 1,2,3oxathiazolidine-3-carboxylate 2,2-dioxide (456 mg, $2.00 \mathrm{mmol}$ ) in DMF (3 mL) was treated with DIPEA (0.89 mL, $5.10 \mathrm{mmol})$ and stirred at rt for $2 \mathrm{~h}$. The RM was adsorbed on Isolute ${ }^{\mathrm{TM}}$ and purified by flash column chromatography on RP18 silica (0.1\% TFA in water:MeCN from 19:1 to 0:1) to afford title compound S-1 (398 mg, 71\%) as a colorless powder. MS (ESI+): m/z = +320.3. ${ }^{1} \mathrm{H}$ NMR (400 MHz, DMSO-d $): \delta 8.02-7.94$ (m, 2H), 7.15-7.07 (m, 2H), 7.03 (t, $\left.J=6.0 \mathrm{~Hz}, 1 \mathrm{H}\right)$, 4.71 (t, $J=5.7 \mathrm{~Hz}, 2 \mathrm{H}), 3.83$ (s, 3H), 3.48 (q, $J=5.9 \mathrm{~Hz}, 2 \mathrm{H}), 1.31$ (s, 9H).

\section{2-(5-(4-Methoxyphenyl)-2H-tetrazol-2-yl)ethan-1-amine (2)}

A solution of S-1 (330 mg, $1.00 \mathrm{mmol})$ in DCM (5 mL) was treated with TFA (1.6 mL, $21.0 \mathrm{mmol})$ and kept at rt for $1 \mathrm{~h}$. The RM was concentrated $i$. vac., adsorbed on Isolute ${ }^{\mathrm{TM}}$ and purified by flash column chromatography on RP18 silica (0.1\% TFA in water:MeCN from 19:1 to 0:1) to afford the amine 2 (319 mg, TFA-salt, 91\%) as a colorless powder. ${ }^{1} \mathrm{H}$ NMR (400 MHz, DMSO- $\left.\mathrm{d}_{6}\right): \delta 8.10$ (s, br, 2H), 8.06-7.98 (m, 2H), 7.17-7.09 (m, 2H), 4.97 (t, $J=5.8$ Hz, 2H), 3.84 (s, 3H), 3.50 (t, $J=5.8 \mathrm{~Hz}, 2 \mathrm{H})$. HRMS (ESI+): m/z calcd for $\mathrm{C}_{10} \mathrm{H}_{14} \mathrm{~N}_{5} \mathrm{O}[\mathrm{M}+\mathrm{H}]^{+}, 220.11929$; found: 220.11935.

\section{5-(4-(p-Tolyloxy)phenyl)-2H-tetrazole (S-2)}

Prepared in analogy to tetrazole 27: To a solution of 4-(p-tolyloxy)benzonitrile (800 mg, $3.80 \mathrm{mmol})$ in dry toluene $(5 \mathrm{~mL})$ were added dibutylstannanone $(95.0 \mathrm{mg}, 0.382 \mathrm{mmol})$ and azidotrimethylsilane $(1.0 \mathrm{~mL}, 7.60 \mathrm{mmol})$. The $\mathrm{RM}$ was heated in a sealed vial to $90^{\circ} \mathrm{C}$ for $18 \mathrm{~h}$. After cooling to rt, the heterogeneous RM was diluted with $\mathrm{MeOH}(20 \mathrm{~mL})$ and concentrated i. vac.. The crude was suspended in $\mathrm{MeOH}(10 \mathrm{~mL})$ and filtered. The solids were washed with heptane $(10 \mathrm{~mL})$ and pentane $(10 \mathrm{~mL})$ and dried $i$. vac. to afford tetrazole S-2 (437 mg, 45\%) as a colorless powder. MS (ESI+/-): m/z = +253.3/-251.2. ${ }^{1} \mathrm{H}$ NMR (400 MHz, DMSO-d 6 ): $\delta 16.74$ (s, br, 1H), 8.23-7.98 (m, 2H), 7.26 (d, $J=8.2 \mathrm{~Hz}, 2 \mathrm{H}), 7.20-7.10$ (m, 2H), 7.07-6.99 (m, 2H), 2.29 $(\mathrm{s}, 3 \mathrm{H})$. 
tert-Butyl (2-(5-(4-(p-tolyloxy)phenyl)-2H-tetrazol-2-yl)ethyl)carbamate (S-3)

Protected amine S-3 was prepared in analogy to S-1 from S-2 (300 mg, $1.19 \mathrm{mmol}$ ) and obtained as a colorless powder (303 mg, 63\%). MS (ESI+): m/z = +396.4. ${ }^{1} \mathrm{H}$ NMR (400 MHz, DMSO-d 6 ): $\delta$ 8.07-8.00 (m, 2H), 7.25 (d, $J=8.3 \mathrm{~Hz}, 2 \mathrm{H}), 7.14-6.98$ (m, 5H), 4.72 (t, $J=5.7 \mathrm{~Hz}, 2 \mathrm{H}), 3.48$ (q, $J=5.8 \mathrm{~Hz}, 2 \mathrm{H}), 2.32$ (s, 3H), 1.31 (s, 9H).

\section{2-(5-(4-(p-Tolyloxy)phenyl)-2H-tetrazol-2-yl)ethan-1-amine (3)}

Amine 3 was prepared in analogy to 2 from S-3 (240 mg, $0.610 \mathrm{mmol})$ and obtained as a colorless powder (243 mg, TFA-salt, 96\%). ${ }^{1} \mathrm{H}$ NMR (400 MHz, DMSO-d $)$ ): $\delta$ 8.12-7.98 (m, 5H), 7.26 (d, $J=8.2 \mathrm{~Hz}, 2 \mathrm{H}), 7.16-7.08$ (m, 2H), 7.06-6.98 (m, 2H), 4.98 (t, $J=5.8 \mathrm{~Hz}, 2 \mathrm{H}), 3.50$ (t, $J=5.8 \mathrm{~Hz}$, 2H), 2.32 (s, 3H). HRMS (ESI+): m/z calcd for $\mathrm{C}_{16} \mathrm{H}_{18} \mathrm{~N}_{5} \mathrm{O}[\mathrm{M}+\mathrm{H}]^{+}, 296.15059$; found: 296.15070.

\section{5-(4-(4-Chlorophenoxy)phenyl)-2H-tetrazole (S-4)}

Tetrazole S-4 was prepared in analogy to S-2 from 4-(4-chlorophenoxy)benzonitrile (1.43 g, $6.23 \mathrm{mmol}$ ) and obtained as a colorless powder (1.49 g, 86\%). ${ }^{1} \mathrm{H}$ NMR (400 MHz, DMSO-d 6 ): $\delta 16.82$ (s, br, 1H), 8.14-8.02 (m, 2H), 7.55-7.46 (m, 2H), 7.27-7.12 (m, 4H). MS (ESI+/-): $\mathrm{m} / \mathrm{z}=+273.0 /-271.2 .{ }^{1} \mathrm{H}$ NMR (400 MHz, DMSO-d 6 ): $\delta 16.74$ (s, br, 1H), 8.23-7.98 (m, 2H), 7.26 (d, $J=8.2 \mathrm{~Hz}, 2 \mathrm{H}), 7.20-7.10$ (m, 2H), 7.07-6.99 (m, 2H), 2.29 (s, 3H).

\section{2-(5-(4-(4-Chlorophenoxy)phenyl)-2H-tetrazol-2-yl)ethan-1-amine (4)}

Step 1. To a solution of tetrazole S-4 (400 mg, $1.47 \mathrm{mmol})$, tert-butyl (2-hydroxyethyl)carbamate (473 mg, $2.93 \mathrm{mmol}$ ) and $\mathrm{PPh}_{3}(577 \mathrm{mg}, 2.20 \mathrm{mmol}$ ) in THF (3 mL) was added di-tert-butyl azodicarboxylate (507 mg, $2.20 \mathrm{mmol}$ ). After stirring at $\mathrm{rt}$ for $3 \mathrm{~h}$, all volatiles were removed $i$. vac. S-5: MS (ESI+): m/z = +416.2.

Step 2. The crude intermediate S-5 was taken up in DCM (5 mL) and TFA (5 mL, $64.9 \mathrm{mmol})$ and kept at rt for $2 \mathrm{~h}$. The RM was concentrated $i$. vac., adsorbed on Isolute ${ }^{\mathrm{TM}}$ and purified by flash column chromatography on RP18 silica $\left[\left(\mathrm{NH}_{4}\right)_{2} \mathrm{CO}_{3} 0.8 \mathrm{~g} / \mathrm{L}\right.$ in water:MeCN from 9:1 to 0:1] to afford title compound 4 (350 mg, 74\%) as a colorless powder. ${ }^{1} \mathrm{H}$ NMR (400 MHz, DMSO-d 6 ): $\delta 8.12-8.03(\mathrm{~m}, 2 \mathrm{H}), 7.54-7.45(\mathrm{~m}, 2 \mathrm{H}), 7.22-7.11(\mathrm{~m}, 4 \mathrm{H}), 4.68(\mathrm{t}, J=6.0 \mathrm{~Hz}, 2 \mathrm{H}), 3.13(\mathrm{t}, J=$ 6.1 Hz, 2H). HRMS (ESI+): m/z calcd for $\mathrm{C}_{15} \mathrm{H}_{14} \mathrm{ClN}_{5} \mathrm{O}[\mathrm{M}+\mathrm{H}]^{+}$, 316.09596; found: 316.09598. 
tert-Butyl (3-(5-(4-(4-chlorophenoxy)phenyl)-2H-tetrazol-2-yl)propyl)carbamate (S-6)

Tetrazole S-4 (210 mg, $0.770 \mathrm{mmol}$ ) was alkylated with tert-butyl 1,2,3-oxathiazinane-3carboxylate 2,2-dioxide (219 $\mathrm{mg}, 0.924 \mathrm{mmol}$ ) in analogy to S-1 affording title compound S-6 (275 mg, 81\%) as a colorless powder. MS (ESI+): m/z = +430.4. ${ }^{1} \mathrm{H}$ NMR (400 MHz, DMSO-d 6 ): $\delta$ 8.11-8.03 (m, 2H), 7.53-7.45 (m, 2H), 7.22-7.10 (m, 4H), 6.95 (s, 1H), 4.72 (t, J = 7.0 Hz, 2H), 3.01 (q, $J=6.5 \mathrm{~Hz}, 2 \mathrm{H}), 2.09$ (q, $J=7.1 \mathrm{~Hz}, 2 \mathrm{H}), 1.36$ (s, 9H).

\section{3-(5-(4-(4-Chlorophenoxy)phenyl)-2H-tetrazol-2-yl)propan-1-amine (5)}

S-6 (200 mg, $0.465 \mathrm{mmol}$ ) was deprotected in analogy to 2 to afford the TFA-salt of 5 (184 mg, 87\%) as a colorless powder. ${ }^{1} \mathrm{H}$ NMR (400 MHz, DMSO-d 6 ): $\delta 8.11-8.04$ (m, 2H), 7.88 (s, 3H), 7.53-7.46 (m, 2H), 7.23-7.12 (m, 4H), 4.85 (t, $J=6.9$ Hz, 2H), 2.97-2.89 (m, 2H), 2.26 (q, $J=$ $7.1 \mathrm{~Hz}, 2 \mathrm{H})$. HRMS (ESI+): m/z calcd for $\mathrm{C}_{16} \mathrm{H}_{16} \mathrm{ClN}_{5} \mathrm{O}[\mathrm{M}+\mathrm{H}]^{+}, 330.11161$; found: 330.11166.

\section{N-(2-(5-(4-(4-chlorophenoxy)phenyl)-2H-tetrazol-2-yl)ethyl)acetamide (6)}

A mixture of 4 (60.0 mg, $0.190 \mathrm{mmol})$ in DCM $(1 \mathrm{~mL})$ and NEt3 $(29 \mu \mathrm{L}, 0.209 \mathrm{mmol})$ was treated with acetic anhydride (20 $\mu \mathrm{L}, 0.209 \mathrm{mmol})$ and stirred at rt for $18 \mathrm{~h}$. The RM was concentrated i. vac., adsorbed on Isolute ${ }^{\mathrm{TM}}$ and purified by flash column chromatography (12 g $\mathrm{SiO}_{2}$, Heptane to EtOAc:MeOH 9:1) to afford title compound 6 (55.0 mg, 79\%) as a colorless powder. ${ }^{1} \mathrm{H}$ NMR (400 MHz, DMSO-d 6 ): $\delta$ 8.12-8.01 (m, 3H), 7.54-7.45 (m, 2H), 7.23-7.11 (m, 4H), 4.79-4.71 (m, 2H), 3.60 (dt, $J=11.9,6.0 \mathrm{~Hz}, 2 \mathrm{H}), 1.76(\mathrm{~s}, 3 \mathrm{H})$. HRMS (ESI+): $m / z$ calcd for $\mathrm{C}_{17} \mathrm{H}_{16} \mathrm{ClN}_{5} \mathrm{O}_{2}$ $[\mathrm{M}+\mathrm{H}]^{+}$, 358.10653; found: 358.10648.

\section{(R)-2-(5-(4-(4-Chlorophenoxy)phenyl)-2H-tetrazol-2-yl)propan-1-amine (7)}

Amine 7 was prepared from S-4 (130 mg, $0.477 \mathrm{mmol})$ and N-Boc-(S)-1-amino-2-propanol (125 mg, $0.715 \mathrm{mmol}$ ) in analogy to 4 via intermediate S-7 [MS (ESI+): m/z = +430.2] and obtained as a brown oil (107 mg, 67\%, two steps). ${ }^{1} \mathrm{H}$ NMR (400 MHz, DMSO-d 6 ): $\delta$ 8.12-8.03 (m, 2H), 7.54-7.45 (m, 2H), 7.22-7.10 (m, 4H), 4.99-4.87 (m, 1H), 3.12-2.98 (m, 2H), 1.55 (d, $J=6.8 \mathrm{~Hz}, 3 \mathrm{H})$. HRMS (ESI+): $\mathrm{m} / \mathrm{z}$ calcd for $\mathrm{C}_{16} \mathrm{H}_{16} \mathrm{ClN}_{5} \mathrm{O}[\mathrm{M}+\mathrm{H}]^{+}$, 330.11161; found: 330.11160. 


\section{(R)-1-(5-(4-(4-Chlorophenoxy)phenyl)-2H-tetrazol-2-yl)propan-2-amine (8)}

Amine 8 was prepared from S-4 (150 mg, $0.550 \mathrm{mmol}$ ) and Boc-D-Alaninol (193 mg, $1.10 \mathrm{mmol}$ ) in analogy to 4 via intermediate S-8 [MS (ESI+): $\mathrm{m} / \mathrm{z}=+430.2$ ] and obtained as a beige powder (144 mg, 78\%, two steps). ${ }^{1} \mathrm{H}$ NMR (400 MHz, DMSO- $\mathrm{d}_{6}$ ): $\delta$ 8.12-8.04 (m, 2H), 7.54-7.45 (m, 2H), 7.22-7.11 (m, 4H), 4.56 (d, $J=6.5 \mathrm{~Hz}, 2 \mathrm{H}), 3.42-3.34$ (m, 1H), 1.04 (d, $J=6.4 \mathrm{~Hz}, 3 \mathrm{H}$ ). HRMS (ESI+): m/z calcd for $\mathrm{C}_{16} \mathrm{H}_{16} \mathrm{ClN}_{5} \mathrm{O}[\mathrm{M}+\mathrm{H}]^{+}$, 330.11161; found: 330.11176.

\section{4-(5-(4-(4-Chlorophenoxy)phenyl)-2H-tetrazol-2-yl)butanoic acid (9)}

A suspension of S-4 $(75.0 \mathrm{mg}, 0.226 \mathrm{mmol}), \mathrm{K}_{2} \mathrm{CO}_{3}(62.3 \mathrm{mg}, 0.451 \mathrm{mmol})$ and ethyl 4-bromobutanoate (36 $\mu \mathrm{L}, 0.271 \mathrm{mmol})$ in DMF (1 mL) was stirred at rt for $18 \mathrm{~h}$. LiOH (1M, $2.26 \mathrm{~mL}, 2.26 \mathrm{mmol}$ ) was added and the RM was stirred at rt for additional $2 \mathrm{~h}$. The RM was partitioned between $2 \mathrm{~N} \mathrm{HCl}(30 \mathrm{~mL})$ and $\mathrm{DCM}(30 \mathrm{~mL})$. The aqueous layer was extracted with DCM $(2 \times 30 \mathrm{~mL})$ and the combined organic phases were dried over $\mathrm{MgSO}_{4}$, filtered and concentrated i. vac. Purification by SFC afforded title compound 9 (43.3 mg, 52\%) as a colorless powder. ${ }^{1} \mathrm{H}$ NMR (400 MHz, DMSO-d ${ }_{6}$ ): $\delta 12.27$ (s, br, $1 \mathrm{H}$ ), 8.12-8.04 (m, 2H), 7.54-7.45 (m, 2H), 7.22-7.11 (m, 4H), $4.76(\mathrm{t}, J=6.9 \mathrm{~Hz}, 2 \mathrm{H}), 2.34(\mathrm{t}, J=7.1 \mathrm{~Hz}, 2 \mathrm{H}), 2.21-2.12(\mathrm{~m}, 2 \mathrm{H})$. HRMS (ESI+): m/z calcd for $\mathrm{C}_{17} \mathrm{H}_{15} \mathrm{ClN}_{4} \mathrm{O}_{3}[\mathrm{M}+\mathrm{H}]^{+}$, 359.09055; found: 359.09042 .

\section{3-(5-(4-(4-Chlorophenoxy)phenyl)-2H-tetrazol-2-yl)propanoic acid (10)}

Acid 10 was prepared in analogy to 9 from S-4 $(75.0 \mathrm{mg}, 0.226 \mathrm{mmol}$ ) and methyl 3-bromopropanoate (36 $\mu \mathrm{L}, 0.271 \mathrm{mmol}$ ) affording title compound $10(5.9 \mathrm{mg}, 7 \%)$ as a colorless powder. ${ }^{1} \mathrm{H}$ NMR (400 MHz, DMSO-d 6 ): $\delta 8.10-8.02$ (m, 2H), 7.54-7.45 (m, 2H), 7.22-7.11 (m, 4H), 4.89 (t, $J=6.5 \mathrm{~Hz}, 2 \mathrm{H}$ ), 3.05 (t, $J=6.5 \mathrm{~Hz}, 2 \mathrm{H}$ ). HRMS (ESI+): m/z calcd for $\mathrm{C}_{17} \mathrm{H}_{15} \mathrm{ClN}_{4} \mathrm{O}_{3}$ $[\mathrm{M}+\mathrm{H}]^{+}, 345.07490$ found: 345.07513 .

\section{Methyl (S)-2-amino-3-(5-(4-(4-chlorophenoxy)phenyl)-2H-tetrazol-2-yl)propanoate (S-9)}

S-9 was prepared from S-4 (120 mg, $0.440 \mathrm{mmol})$ and Boc-Ser-OMe (241 mg, $1.10 \mathrm{mmol})$ in analogy to 4 and obtained as a colorless powder (125 mg, 63\%, two steps). MS (ESI+/-): m/z = +374.1/-372.0. ${ }^{1} \mathrm{H}$ NMR (400 MHz, DMSO-d 6 ): $\delta 8.72$ (s, br, 3H), 8.12-8.04 (m, 2H), 7.55-7.46 (m, 2H), 7.25-7.11 (m, 4H), 5.26 (dd, $J=4.7,1.6 \mathrm{~Hz}, 2 \mathrm{H}), 4.91$ (t, $J=4.7 \mathrm{~Hz}, 1 \mathrm{H}$ ), 3.83 (s, 3H). 


\section{(S)-2-Amino-3-(5-(4-(4-chlorophenoxy)phenyl)-2H-tetrazol-2-yl)propanoic acid (11)}

A solution of S-9 (80.0 mg, $0.164 \mathrm{mmol})$ in $\mathrm{MeOH}(8 \mathrm{~mL})$ was treated with $\mathrm{LiOH}(1 \mathrm{M}, 1.64 \mathrm{~mL}$, $1.64 \mathrm{mmol}$ ) and stirred at $\mathrm{rt}$ for $90 \mathrm{~min}$. The RM was concentrated i. vac. and purified by preparative HPLC affording title compound 11 (5.5 mg, 7\%) as a colorless powder. ${ }^{1} \mathrm{H}$ NMR (400 MHz, DMSO-d $\left.\mathrm{d}_{6}\right): \delta 8.14-8.06$ (m, 2H), 7.80 (s, br, 2H), 7.54-7.45 (m, 2H), 7.23-7.11 (m, 4H), 5.15 (dd, $J=14.6,3.3 \mathrm{~Hz}, 1 \mathrm{H}$ ), 4.93 (dd, $J=14.6,9.1 \mathrm{~Hz}, 1 \mathrm{H}$ ), 3.89 (dd, $J=9.2,3.3 \mathrm{~Hz}$, 1H). HRMS (ESI+): m/z calcd for $\mathrm{C}_{16} \mathrm{H}_{14} \mathrm{ClN}_{5} \mathrm{O}_{3}[\mathrm{M}+\mathrm{H}]^{+}, 360.08579$ found: 360.08560 .

\section{Methyl (2-(5-(4-(4-chlorophenoxy)phenyl)-2H-tetrazol-2-yl)ethyl)glycinate (S-10)}

A suspension of $4(150 \mathrm{mg}, 0.475 \mathrm{mmol}), \mathrm{Cs}_{2} \mathrm{CO}_{3}(310 \mathrm{mg}, 0.950 \mathrm{mmol})$ and methyl 2-bromoacetate ( $87 \mu \mathrm{L}, 0.950 \mathrm{mmol}$ ) in MeCN (2 mL) was stirred at rt for $3 \mathrm{~h}$. The RM was filtered over a pad of Celite ${ }^{\mathrm{TM}}$ and eluted with additional EtOAc. Purification by flash column chromatography (24 g SiO 2 , Heptane to EtOAc) afforded title compound S-10 (58 mg, 30\%) as a beige powder. MS (ESI+): $\mathrm{m} / \mathrm{z}=+388.1 .{ }^{1} \mathrm{H}$ NMR (400 MHz, DMSO-d 6 ): $\delta 8.11-8.03$ (m, 2H), 7.53-7.45 (m, 2H), 7.22-7.11 (m, 4H), 4.77 (dd, $J=6.7,5.5$ Hz, 2H), 3.60 (s, 3H), 3.42 (s, 2H), 3.36 (s, 2H), 3.16 (t, $J=6.1 \mathrm{~Hz}, 2 \mathrm{H})$.

\section{(2-(5-(4-(4-Chlorophenoxy)phenyl)-2H-tetrazol-2-yl)ethyl)glycine (12)}

A solution of S-10 $(58.0 \mathrm{mg}, 0.150 \mathrm{mmol})$ in THF $(1.4 \mathrm{~mL})$ was treated with water $(0.2 \mathrm{~mL})$ and $\mathrm{LiOH} \times \mathrm{H}_{2} \mathrm{O}(20.1 \mathrm{mg}, 0.480 \mathrm{mmol})$. The $\mathrm{RM}$ was heated to $60^{\circ} \mathrm{C}$ for $30 \mathrm{~min}$, and concentrated i. vac.. The residue was suspended in a small amount of water and acidified with $2 \mathrm{~N} \mathrm{HCl}$ to $\mathrm{pH} 4$. The resulting suspension was filtered and the solids collected were washed with several portions of water. Drying i. vac. afforded title compound $12(28.0 \mathrm{mg}, 46 \%)$ as a colorless powder. ${ }^{1} \mathrm{H}$ NMR (400 MHz, DMSO-d 6 ): $\delta$ 8.12-8.04 (m, 2H), 7.53-7.45 (m, 2H), 7.22-7.11 (m, 4H), 4.82 (t, $J=$ $6.0 \mathrm{~Hz}, 2 \mathrm{H}$ ), 3.25 (s, 2H), 3.21 (t, $J=6.0 \mathrm{~Hz}, 2 \mathrm{H})$. HRMS (ESI+): m/z calcd for $\mathrm{C}_{17} \mathrm{H}_{16} \mathrm{ClN}_{5} \mathrm{O}_{3}$ $[\mathrm{M}+\mathrm{H}]^{+}, 347.10144$ found: 374.10141. 
tert-Butyl (S)-3-((tert-butoxycarbonyl)amino)-4-(5-(4-(4-chlorophenoxy)phenyl)-2Htetrazol-2-yl)butanoate [(S)-S-11]

Intermediate (S)-S-11 was prepared in analogy to $(\boldsymbol{S})$-30 from S-4 $(200 \mathrm{mg}, 0.733 \mathrm{mmol})$ and (S)-29 (330 mg, $0.880 \mathrm{mmol}$ ) and obtained as a colorless semi-solid (395 mg, 97\%). MS (ESI+): $\mathrm{m} / \mathrm{z}=+530.2 .{ }^{1} \mathrm{H}$ NMR $\left(400 \mathrm{MHz}, \mathrm{CD}_{3} \mathrm{OD}\right): \delta 8.11$ (d, $\left.J=8.4 \mathrm{~Hz}, 2 \mathrm{H}\right), 7.44-7.36(\mathrm{~m}, 2 \mathrm{H}), 7.12$ (d, $J=8.5 \mathrm{~Hz}, 2 \mathrm{H}$ ), 7.09-7.02 (m, 2H), 4.91-4.85 (m, 1H), 4.74 (dd, $J=13.6,8.1 \mathrm{~Hz}, 1 \mathrm{H}$ ), 4.47 (tt, $J=8.3,5.1 \mathrm{~Hz}, 1 \mathrm{H}$ ), 2.64 (dd, $J=15.7,6.0 \mathrm{~Hz}, 1 \mathrm{H}$ ), 2.51 (dd, $J=15.8,8.4 \mathrm{~Hz}, 1 \mathrm{H}$ ), 1.46 (s, 9H), 1.32 (s, 9H).

(S)-3-Amino-4-(5-(4-(4-chlorophenoxy)phenyl)-2H-tetrazol-2-yl)butanoic acid [(S)-13]

(S)-S-11 (395 $\mathrm{mg}, 0.746 \mathrm{mmol}$ ) was deprotected in analogy to (S)-22 to afford the hydrochloride of (S)-13 (238 mg, 76\%) as a colorless powder. ${ }^{1} \mathrm{H}$ NMR (400 MHz, $\mathrm{CD}_{3} \mathrm{OD}$ ): $\delta$ 8.22-8.12 (m, 2H), 7.47-7.39 (m, 2H), 7.20-7.12 (m, 2H), 7.12-7.05 (m, 2H), 5.14 (d, J = 5.5 Hz, 2H), 4.28 (dq, $J=7.1,5.6 \mathrm{~Hz}, 1 \mathrm{H}), 2.95$ (dd, $J=17.9,5.7 \mathrm{~Hz}, 1 \mathrm{H}), 2.78$ (dd, $J=17.9,7.0 \mathrm{~Hz}, 1 \mathrm{H})$. HRMS (ESI+): $\mathrm{m} / \mathrm{z}$ calcd for $\mathrm{C}_{17} \mathrm{H}_{16} \mathrm{ClN}_{5} \mathrm{O}_{3}[\mathrm{M}+\mathrm{H}]^{+}, 374.10144$ found: 374.10141 .

tert-Butyl (R)-3-((tert-butoxycarbonyl)amino)-4-(5-(4-(4-chlorophenoxy)phenyl)-2Htetrazol-2-yl)butanoate [(R)-S-11]

A clear yellow solution of $\mathrm{PPh}_{3}(2.50 \mathrm{~g}, 9.53 \mathrm{mmol})$ and DIAD $(1.85 \mathrm{~mL}, 9.53 \mathrm{mmol})$ in THF $(10 \mathrm{~mL})$ was added to a solution of tetrazole S-4 $(2.00 \mathrm{~g}, 7.33 \mathrm{mmol})$ and $(R)$-tert-butyl 3-((tertbutoxycarbonyl)amino)-4-hydroxybutanoate $(2.02 \mathrm{~g}, 7.33 \mathrm{mmol})$ in THF $(10 \mathrm{~mL})$ at $0^{\circ} \mathrm{C}$. The RM was stirred for $90 \mathrm{~min}$ at $\mathrm{rt}$ and concentrated i. vac. affording crude (R)-S-11 [MS (ESI+): $\mathrm{m} / \mathrm{z}=+530.2]$, which was used directly in the next step.

\section{(R)-3-Amino-4-(5-(4-(4-chlorophenoxy)phenyl)-2H-tetrazol-2-yl)butanoic acid [(R)-13]}

Crude (R)-S-11 was dissolved in 4M HCl in dioxane $(40.0 \mathrm{~mL}, 160 \mathrm{mmol})$ and heated to $80^{\circ} \mathrm{C}$ for $3 \mathrm{~h}$. The resulting colorless suspension was filtered, washed with DCM, and dried i. vac. to afford (R)-13 (1.68 g, 55\%, two steps) as a colorless powder. ${ }^{1} \mathrm{H}$ NMR (400 MHz, DMSO-d 6 ): $\delta 8.36$ (s, br, 2H), 8.15-8.06 (m, 2H), 7.55-7.46 (m, 2H), 7.25-7.19 (m, 2H), 7.19-7.11 (m, 2H), 5.06 (d, $J$ 
= $5.7 \mathrm{~Hz}, 2 \mathrm{H}), 4.12-4.03(\mathrm{~m}, 1 \mathrm{H}), 2.79$ (dd, $J=6.5,2.2 \mathrm{~Hz}, 2 \mathrm{H})$. HRMS (ESI+): m/z calcd for $\mathrm{C}_{17} \mathrm{H}_{16} \mathrm{ClN}_{5} \mathrm{O}_{3}[\mathrm{M}+\mathrm{H}]^{+}, 374.10144$ found: 374.10147.

\section{5-(4-(4-Fluorophenoxy)phenyl)-2H-tetrazole (S-12)}

Tetrazole S-12 was prepared in analogy to 27 from 4-(4-fluorophenoxy)benzonitrile (4.00 g, $18.8 \mathrm{mmol}$ ) and obtained as a colorless powder (3.80 g, 79\%). MS (ESI+/-): $\mathrm{m} / \mathrm{z}=+257.1 /-255.1$. ${ }^{1} \mathrm{H}$ NMR (400 MHz, DMSO-d 6 ): $\delta 16.79$ (s, br, 1H), 8.08-8.00 (m, 2H), 7.36-7.25 (m, 2H), 7.25$7.13(\mathrm{~m}, 4 \mathrm{H}) .{ }^{19} \mathrm{~F}$ NMR (376 MHz, DMSO-d $): \delta-118.56$ (s, 1F).

tert-Butyl (R)-3-((tert-butoxycarbonyl)amino)-4-(5-(4-(4-fluorophenoxy)phenyl)-2Htetrazol-2-yl)butanoate [(R)-S-13]

Intermediate $(\boldsymbol{R})$-S-13 was prepared in analogy to $(\boldsymbol{S})$-30 from $\mathbf{S - 1 2}(350 \mathrm{mg}, 1.37 \mathrm{mmol})$ and (R)-29 (553 mg, $1.64 \mathrm{mmol}$ ) and obtained as a colorless solid (551 mg, 79\%) in ca. 88\% purity. The material was carried forward without additional purification. MS (ESI+): $\mathrm{m} / \mathrm{z}=+514.2$.

\section{(R)-3-amino-4-(5-(4-(4-fluorophenoxy)phenyl)-2H-tetrazol-2-yl)butanoic acid [(R)-14]}

(R)-S-13 (550 mg, ca. 88\%) was deprotected in analogy to (S)-22 to afford the hydrochloride of (R)-14 (316 mg, 73\%, two steps) as a colorless powder. ${ }^{1} \mathrm{H}$ NMR (400 MHz, DMSO-d6): $\delta 8.22$ (s, br, 2H), 8.12-8.06 (m, 2H), 7.35-7.25 (m, 2H), 7.24-7.12 (m, 4H), 5.04 (d, $J=5.8 \mathrm{~Hz}, 2 \mathrm{H}$ ), 4.08 (q, $J=6.1 \mathrm{~Hz}, 1 \mathrm{H}), 2.78$ (td, $J=17.4,6.5 \mathrm{~Hz}, 2 \mathrm{H}) .{ }^{19} \mathrm{~F}$ NMR (376 MHz, DMSO-d $): \delta$ 120.88-(-120.97) (m, 1F). HRMS (ESI+): m/z calcd for $\mathrm{C}_{17} \mathrm{H}_{16} \mathrm{FN}_{5} \mathrm{O}_{3}[\mathrm{M}+\mathrm{H}]^{+}$, 358.13099 found: 358.13104 .

\section{4-(Pentyloxy)benzonitrile (S-14)}

A suspension of 4-hydroxybenzonitrile $(5.00 \mathrm{~g}, 42.0 \mathrm{mmol})$, 1-bromopentane (6.25 $\mathrm{mL}$, $50.4 \mathrm{mmol})$ and $\mathrm{K}_{2} \mathrm{CO}_{3}(8.70 \mathrm{~g}, 63.0 \mathrm{mmol})$ in DMF $(15 \mathrm{~mL})$ was stirred at $\mathrm{rt}$ for $72 \mathrm{~h}$. The RM was diluted with EtOAc:Heptane $(1: 1,150 \mathrm{~mL})$, washed with water $(2 \times 100 \mathrm{~mL})$ and brine (50 mL), dried over $\mathrm{Na}_{2} \mathrm{SO}_{4}$, filtered and concentrated i. vac.. The crude was adsorbed on Isolute ${ }^{\mathrm{TM}}$ and purified by flash column chromatography ( $80 \mathrm{~g} \mathrm{SiO}_{2}$, TBME in heptane, 0 to 25\%) to afford 
title compound S-14 (4.80 mg, 59\%) as a colorless oil. MS (ESI+): m/z = +190.1. ${ }^{1} \mathrm{H}$ NMR (400 MHz, DMSO-d $): \delta$ 7.79-7.71 (m, 2H), 7.14-7.05 (m, 2H), 4.05 (t, $J=6.5$ Hz, 2H), 1.781.67 (m, 2H), 1.45-1.27 (m, 4H), 0.89 (t, $J=7.0 \mathrm{~Hz}, 3 \mathrm{H})$.

\section{5-(4-(Pentyloxy)phenyl)-2H-tetrazole (S-15)}

Tetrazole S-15 was prepared in analogy to 27 from nitrile S-14 (1.14 g, $6.00 \mathrm{mmol})$ and obtained as a beige powder (1.03 g, 72\%). MS (ESI+/-): m/z = +233.5/-231.2. ${ }^{1} \mathrm{H}$ NMR (400 MHz, CD $\left.{ }_{3} \mathrm{OD}\right)$ : $\delta$ 7.97-7.89 (m, 2H), 7.14-7.07 (m, 2H), 4.06 (t, $J=6.4$ Hz, 2H), 1.87-1.76 (m, 2H), 1.55-1.35 (m, 4H), 0.96 (t, $J=7.1 \mathrm{~Hz}, 3 \mathrm{H})$.

tert-Butyl (R)-3-((tert-butoxycarbonyl)amino)-4-(5-(4-(pentyloxy)phenyl)-2H-tetrazol-2yl)butanoate [(R)-S-16]

Tetrazole S-15 (116 mg, $0.500 \mathrm{mmol}$ ) was alkylated in analogy to $(\boldsymbol{S})$-30 using reagent $(\boldsymbol{R})-\mathbf{2 9}$ (202 mg, $0.600 \mathrm{mmol}$ ). Flash column chromatography on RP18 silica (0.1\% TFA in water:MeCN from 19:1 to 0:1) afforded title compound (R)-S-16 (208 $\mathrm{mg}, 82 \%)$ as a colorless viscous oil. MS (ESI+): $\mathrm{m} / \mathrm{z}=+490.3$.

(R)-3-Amino-4-(5-(4-(pentyloxy)phenyl)-2H-tetrazol-2-yl)butanoic acid [(R)-15]

(R)-S-16 (208 mg, $0.425 \mathrm{mmol}$ ) was deprotected in analogy to $(\boldsymbol{S})$-22 to afford the hydrochloride of (R)-15 (139 mg, 87\%) as a colorless powder. ${ }^{1} \mathrm{H}$ NMR (400 MHz, $\left.\mathrm{CD}_{3} \mathrm{OD}\right): \delta$ 8.12-8.01 (m, 2H), 7.11-7.03 (m, 2H), 5.11 (d, $J=5.5 \mathrm{~Hz}, 2 \mathrm{H}), 4.26$ (dq, $J=7.1,5.6 \mathrm{~Hz}, 1 \mathrm{H}), 4.06(t, J=6.4 \mathrm{~Hz}$, 2H), 2.93 (dd, $J=17.9,5.8 \mathrm{~Hz}, 1 \mathrm{H}), 2.77$ (dd, $J=17.9,7.0 \mathrm{~Hz}, 1 \mathrm{H}), 1.81$ (dt, $J=8.0,6.4 \mathrm{~Hz}, 2 \mathrm{H}$ ), 1.55-1.36 (m, 4H), 0.97 (t, $J=7.1 \mathrm{~Hz}, 3 \mathrm{H})$. HRMS (ESI+): m/z calcd for $\mathrm{C}_{16} \mathrm{H}_{23} \mathrm{~N}_{5} \mathrm{O}_{3}[\mathrm{M}+\mathrm{H}]^{+}$, 334.18737 found: 334.18735.

\section{4-Phenethoxybenzonitrile (S-17)}

Nitrile S-17 was prepared in analogy to S-14 from 4-hydroxybenzonitrile (2.00 g, $16.8 \mathrm{mmol}$ ) and (2-iodoethyl)benzene (3.65 mL, $25.2 \mathrm{mmol}$ ) with heating to $65^{\circ} \mathrm{C}$ and obtained as a yellow liquid (1.29 g, 33\%). MS (ESI+): m/z = +224.1. ${ }^{1} \mathrm{H}$ NMR (400 MHz, $\left.\mathrm{CDCl}_{3}\right): \delta 7.63-7.55$ (m, 2H), $7.40-$ 
7.32 (m, 2H), 7.32-7.24 (m, 3H), 7.00-6.92 (m, 2H), 4.24 (t, $J=7.0 \mathrm{~Hz}, 2 \mathrm{H}), 3.15$ (t, $J=7.0 \mathrm{~Hz}$, $2 \mathrm{H})$.

\section{5-(4-Phenethoxyphenyl)-2H-tetrazole (S-18)}

Tetrazole S-18 was prepared in analogy to $\mathbf{2 7}$ from nitrile S-17 (2.52 g, $11.3 \mathrm{mmol})$ and obtained as a colorless powder (1.45 g, 47\%). MS (ESI+/-): m/z $=+267.1 /-265.1 .{ }^{1} \mathrm{H}$ NMR $(400 \mathrm{MHz}$, DMSO-d $\mathrm{d}_{6}$ ): $\delta 16.60$ (s, br, 1H), 8.00-7.92 (m, 2H), 7.40-7.29 (m, 4H), 7.29-7.19 (m, 1H), 7.19$7.12(\mathrm{~m}, 2 \mathrm{H}), 4.29$ (t, $J=6.9 \mathrm{~Hz}, 2 \mathrm{H}), 3.07$ (t, $J=6.9 \mathrm{~Hz}, 2 \mathrm{H})$.

tert-Butyl (R)-3-((tert-butoxycarbonyl)amino)-4-(5-(4-phenethoxyphenyl)-2H-tetrazol-2yl)butanoate [(R)-S-19]

Tetrazole S-18 (150 mg, $0.563 \mathrm{mmol}$ ) was alkylated in analogy to $(\boldsymbol{S})$-30 using reagent $(\boldsymbol{R})$-29 (228 mg, $0.676 \mathrm{mmol})$. Flash column chromatography on RP18 silica (0.1\% TFA in water:MeCN from 19:1 to 0:1) afforded title compound (R)-S-19 (264 $\mathrm{mg}$, 88\%) as a yellowish viscous oil. MS (ESI +$): \mathrm{m} / \mathrm{z}=+524.4$.

\section{(R)-3-Amino-4-(5-(4-phenethoxyphenyl)-2H-tetrazol-2-yl)butanoic acid [(R)-16]}

(R)-S-19 (264 mg, $0.505 \mathrm{mmol}$ ) was deprotected in analogy to $(\boldsymbol{S})$-22 to afford the hydrochloride of ( $R$ )-16 (173 mg, 83\%) as a colorless powder. ${ }^{1} \mathrm{H}$ NMR (400 MHz, $\mathrm{CD}_{3} \mathrm{OD}$ ): $\delta$ 8.11-8.03 (m, 2H), 7.36-7.26 (m, 4H), 7.22 (td, $J=6.4,6.0,2.5 \mathrm{~Hz}, 1 \mathrm{H}$ ), 7.11-7.03 (m, 2H), 5.10 (d, $J=5.5 \mathrm{~Hz}$, 2H), 4.31-4.21 (m, 3H), 3.11 (t, $J=6.8 \mathrm{~Hz}, 2 \mathrm{H}$ ), 2.92 (dd, $J=17.9,5.8 \mathrm{~Hz}, 1 \mathrm{H}$ ), 2.75 (dd, $J=17.9$, $7.0 \mathrm{~Hz}, 1 \mathrm{H}$ ). HRMS (ESI+): m/z calcd for $\mathrm{C}_{19} \mathrm{H}_{21} \mathrm{~N}_{5} \mathrm{O}_{3}[\mathrm{M}+\mathrm{H}]^{+}, 368.17172$ found: 368.17181 .

\section{(R)-4-((1-Phenylpropan-2-yl)oxy)benzonitrile (S-20)}

A solution of (S)-1-phenyl-2-propanol (2.02 mL, $14.7 \mathrm{mmol}$ ), 4-hydroxybenzonitrile (1.75 g, $14.7 \mathrm{mmol}$ ) and $\mathrm{PPh}_{3}(5.01 \mathrm{~g}, 19.1 \mathrm{mmol})$ in 2-MeTHF (49 mL) was treated with DIAD (3.71 mL, $19.1 \mathrm{mmol}$ ), and stirred at rt for $3 \mathrm{~h}$. The RM was concentrated $i$. vac., adsorbed on Isolute ${ }^{\mathrm{TM}}$ and purified by flash column chromatography on RP18 silica (0.1\% TFA in water:MeCN from 19:1 to 0:1) to afford title compound S-20 (1.99 $\mathrm{g}, 51 \%$ ) as a yellow liquid in ca. 90\% purity. MS (ESI+/-) no ionization. The material was carried forward without additional purification. 


\section{(R)-5-(4-((1-Phenylpropan-2-yl)oxy)phenyl)-2H-tetrazole (S-21)}

Tetrazole S-21 was prepared in analogy to 27 from nitrile S-20 (1.99 g) and obtained after flash column chromatography on RP18 silica (0.1\% TFA in water:MeCN from 19:1 to 0:1) as a colorless solid (1.45 g, 66\%). MS (ESI+/-): m/z = +281.2.1/-279.2. ${ }^{1} \mathrm{H}$ NMR (400 MHz, DMSO-d 6 ): $\delta 16.60$ (s, br, 1H), 7.98-7.90 (m, 2H), 7.29 (d, $J=4.3 \mathrm{~Hz}, 4 \mathrm{H}$ ), 7.25-7.10 (m, 3H), 4.83 (q, $J=6.1 \mathrm{~Hz}$, 1H), 3.01 (dd, $J=13.7,6.5 \mathrm{~Hz}, 1 \mathrm{H}$ ), 2.89 (dd, $J=13.7,5.9 \mathrm{~Hz}, 1 \mathrm{H}$ ), 1.26 (d, $J=6.0 \mathrm{~Hz}, 3 \mathrm{H}$ ).

tert-Butyl (S)-3-((tert-butoxycarbonyl)amino)-4-(5-(4-(((R)-1-phenylpropan-2yl)oxy)phenyl)-2H-tetrazol-2-yl)butanoate [(S)-S-22]

Tetrazole S-21 (165 mg, $0.589 \mathrm{mmol}$ ) was alkylated in analogy to $(\boldsymbol{S})$-30 to afford title compound (S)-S-22 (319 mg, 99\%) as a viscous yellow oil. MS (ESI+): m/z = +538.4.

(S)-3-amino-4-(5-(4-(((R)-1-phenylpropan-2-yl)oxy)phenyl)-2H-tetrazol-2-yl)butanoic acid [(S)-17]

(S)-S-22 (319 $\mathrm{mg}, 0.594 \mathrm{mmol}$ ) was deprotected in analogy to $(\boldsymbol{S})$-22 to afford the hydrochloride of (S)-17 (180 mg, 71\%) as a colorless powder. ${ }^{1} \mathrm{H}$ NMR (400 MHz, $\left.\mathrm{CD}_{3} \mathrm{OD}\right): \delta 8.11-8.00(\mathrm{~m}$, 2H), 7.29 (d, $J=4.3 \mathrm{~Hz}, 4 \mathrm{H}), 7.24-7.17$ (m, 1H), 7.10-7.02 (m, 2H), 5.12 (d, $J=5.5 \mathrm{~Hz}, 2 \mathrm{H}$ ), 4.78 (q, $J=6.1 \mathrm{~Hz}, 1 \mathrm{H}$ ), 4.27 (dq, $J=7.2,5.6 \mathrm{~Hz}, 1 \mathrm{H}$ ), 3.08 (dd, $J=13.7,6.5 \mathrm{~Hz}, 1 \mathrm{H}$ ), 2.99-2.87 (m, 2H), 2.78 (dd, $J=17.9,7.0 \mathrm{~Hz}, 1 \mathrm{H}$ ), 1.34 (d, $J=6.1 \mathrm{~Hz}, 3 \mathrm{H})$. HRMS (ESI+): m/z calcd for $\mathrm{C}_{20} \mathrm{H}_{23} \mathrm{~N}_{5} \mathrm{O}_{3}[\mathrm{M}+\mathrm{H}]^{+}, 382.18737$ found: 382.18759 .

\section{4-((Benzyloxy)methyl)benzonitrile (S-23)}

To a solution of 4-(hydroxymethyl)benzonitrile (5.00 g, $37.6 \mathrm{mmol})$ in DMF (16 mL) were added $\mathrm{KOtBu}(4.64 \mathrm{~g}, 41.3 \mathrm{mmol})$ and benzylbromide $(4.91 \mathrm{~mL}, 41.3 \mathrm{mmol})$ at $0-5^{\circ} \mathrm{C}$. The $\mathrm{RM}$ was allowed to warm to rt and stirred for $2 \mathrm{~h}$, before it was diluted with water $(150 \mathrm{~mL})$ and extracted with EA $(4 \times 50 \mathrm{~mL})$. The combined organic extracts were washed with water $(50 \mathrm{~mL})$ and brine (50 mL), dried over $\mathrm{Na}_{2} \mathrm{SO}_{4}$, filtered and concentrated i. vac.. Purification by flash column chromatography (120 g SiO 2 , Heptane to EtOAc) afforded title compound S-23 (7.10 g, 84\%) as a viscous yellow oil. MS (ESI+/-): no ionization. ${ }^{1} \mathrm{H}$ NMR (400 MHz, $\left.\mathrm{CDCl}_{3}\right): \delta 7.70-7.63(\mathrm{~m}$, 2H), 7.50 (d, $J=8.0 \mathrm{~Hz}, 2 \mathrm{H}), 7.45-7.30$ (m, 5H), 4.63 (d, $J=1.4 \mathrm{~Hz}, 4 \mathrm{H})$. 


\section{5-(4-((Benzyloxy)methyl)phenyl)-2H-tetrazole (S-24)}

Nitrile S-23 (5.50 g, $24.6 \mathrm{mmol}$ ) was converted into tetrazole S-24 as described for S-2 and obtained after flash column chromatography on RP18 silica (0.1\% TFA in water:MeCN from 19:1 to $0: 1$ ) as a colorless powder (5.49 $\mathrm{g}, 80 \%)$. MS (ESI+/-): $\mathrm{m} / \mathrm{z}=+267.2 /-265.2 .{ }^{1} \mathrm{H}$ NMR (400 MHz, DMSO-d $\mathrm{d}_{6}$ ): $\delta 16.87$ (s, br, 1H), 8.07-8.01 (m, 2H), 7.60 (d, $J=8.0 \mathrm{~Hz}, 2 \mathrm{H}$ ), 7.43-7.36 (m, 4H), 7.32 (ddt, $J=8.6,5.6,3.0 \mathrm{~Hz}, 1 \mathrm{H}$ ), 4.64 (s, 2H), 4.59 (s, 2H).

tert-Butyl (S)-4-(5-(4-((benzyloxy)methyl)phenyl)-2H-tetrazol-2-yl)-3-((tertbutoxycarbonyl)amino)butanoate [(S)-S-25]

Tetrazole S-24 (200 mg, $0.751 \mathrm{mmol}$ ) was alkylated in analogy to $(\boldsymbol{S})$-30 to afford title compound (S)-S-25 (387 mg, 94\%) as a viscous yellow oil. MS (ESI+): m/z = +524.2.

(S)-3-Amino-4-(5-(4-((benzyloxy)methyl)phenyl)-2H-tetrazol-2-yl)butanoic acid [(S)-18]

(S)-S-25 (387 mg, $0.739 \mathrm{mmol}$ ) was deprotected in analogy to $(\boldsymbol{S})$-22 to afford the hydrochloride of (S)-18 (237 mg, 78\%) as a colorless powder. ${ }^{1} \mathrm{H}$ NMR (400 MHz, $\left.\mathrm{CD}_{3} \mathrm{OD}\right): \delta 8.19-8.12(\mathrm{~m}$, 2H), 7.58-7.52 (m, 2H), 7.43-7.33 (m, 4H), 7.33-7.25 (m, 1H), 5.14 (d, $J=5.5$ Hz, 2H), 4.64 (s, 2H), 4.61 (s, 2H), 4.27 (dq, $J=7.0,5.6 \mathrm{~Hz}, 1 \mathrm{H}$ ), 2.94 (dd, $J=17.9,5.8 \mathrm{~Hz}, 1 \mathrm{H}$ ), 2.77 (dd, $J=$ 17.9, $7.0 \mathrm{~Hz}, 1 \mathrm{H})$. HRMS (ESI+): $\mathrm{m} / \mathrm{z}$ calcd for $\mathrm{C}_{19} \mathrm{H}_{21} \mathrm{~N}_{5} \mathrm{O}_{3}[\mathrm{M}+\mathrm{H}]^{+}, 368.17172$ found: 368.17181 .

tert-Butyl (R)-4-(5-(4-((benzyloxy)methyl)phenyl)-2H-tetrazol-2-yl)-3-((tertbutoxycarbonyl)amino)butanoate [(R)-S-25]

Tetrazole S-24 (200 mg, $0.751 \mathrm{mmol}$ ) was alkylated in analogy to (S)-30 using (R)-29 (279 mg, $0.826 \mathrm{mmol}$ ) to afford title compound $(\boldsymbol{R})$-S-25 (312 mg, 78\%) as a viscous yellow oil. MS (ESI+): $\mathrm{m} / \mathrm{z}=+524.4$.

(R)-3-Amino-4-(5-(4-((benzyloxy)methyl)phenyl)-2H-tetrazol-2-yl)butanoic acid [(R)-18]

(R)-S-25 (312 mg, $0.596 \mathrm{mmol}$ ) was deprotected in analogy to $(\boldsymbol{S})-22$ to afford the hydrochloride of $(\boldsymbol{R})-18$ (218 mg, 89\%) as a colorless powder. ${ }^{1} \mathrm{H}$ NMR (400 MHz, $\left.\mathrm{CD}_{3} \mathrm{OD}\right): \delta 8.21-8.14(\mathrm{~m}$, 2H), 7.60-7.54 (m, 2H), 7.44-7.35 (m, 4H), 7.35-7.27 (m, 1H), 5.16 (d, J = 5.5 Hz, 2H), 4.66 (s, 
2H), 4.63 (s, 2H), 4.29 (dq, $J=6.9,5.6 \mathrm{~Hz}, 1 \mathrm{H}$ ), 2.96 (dd, $J=17.9,5.8 \mathrm{~Hz}, 1 \mathrm{H}$ ), 2.79 (dd, $J=17.9$, $7.0 \mathrm{~Hz}, 1 \mathrm{H}$ ). HRMS (ESI+): m/z calcd for $\mathrm{C}_{19} \mathrm{H}_{21} \mathrm{~N}_{5} \mathrm{O}_{3}[\mathrm{M}+\mathrm{H}]^{+}, 368.17172$ found: 368.17181 .

\section{4-(4-(Oxazol-2-yl)phenoxy)benzonitrile (S-26)}

A suspension of 4-(oxazol-2-yl)phenol (200 mg, $1.24 \mathrm{mmol}$ ), 4-fluorobenzonitrile (301 mg, $2.48 \mathrm{mmol})$ and $\mathrm{K}_{2} \mathrm{CO}_{3}(515 \mathrm{mg}, 3.72 \mathrm{mmol})$ in $\mathrm{DMF}(1.2 \mathrm{~mL})$ was heated to $100^{\circ} \mathrm{C}$ for $16 \mathrm{~h}$. The RM was concentrated $i$. vac., adsorbed on Isolute ${ }^{\mathrm{TM}}$ and purified by flash column chromatography on RP18 silica (0.1\% TFA in water:MeCN from 19:1 to 0:1) to afford title compound S-26 (295 mg, 86\%) as a colorless powder. MS (ESI+/-): m/z = +263.1/-261.1.

\section{2-(4-(4-(2H-Tetrazol-5-yl)phenoxy)phenyl)oxazole (S-27)}

Nitrile S-26 (295 mg, $1.13 \mathrm{mmol}$ ) was converted into tetrazole S-27 as described for S-2 and obtained after flash column chromatography on RP18 silica (0.1\% TFA in water:MeCN from 19:1 to 0:1) as a colorless powder (280 $\mathrm{mg}, 78 \%$ ). MS (ESI+/-): $\mathrm{m} / \mathrm{z}=+306.1 /-304.1$.

tert-Butyl (R)-3-((tert-butoxycarbonyl)amino)-4-(5-(4-(4-(oxazol-2-yl)phenoxy)phenyl)-2Htetrazol-2-yl)butanoate [(R)-S-28]

Tetrazole S-27 (150 mg, $0.491 \mathrm{mmol}$ ) was alkylated in analogy to $(\boldsymbol{R})$-S-11 to afford title compound ( $\boldsymbol{R})$-S-28 after flash column chromatography on RP18 silica (0.1\% TFA in water:MeCN from 19:1 to 0:1) as a yellow liquid (287 $\mathrm{mg}, 95 \%$ ) in ca. $92 \%$ purity. The material was carried forward without additional purification. MS (ESI+): $\mathrm{m} / \mathrm{z}=+563.4$.

(R)-3-Amino-4-(5-(4-(4-(oxazol-2-yl)phenoxy)phenyl)-2H-tetrazol-2-yl)butanoic acid $[(R)-$ 19]

(R)-S-28 (140 mg, $0.249 \mathrm{mmol}$ ) was deprotected in analogy to $(\boldsymbol{S})$-22 to afford the hydrochloride of $(\boldsymbol{R}) \mathbf{- 1 9}$ (79.7 mg, 71\%) as a colorless powder. ${ }^{1} \mathrm{H}$ NMR (400 MHz, $\left.\mathrm{CD}_{3} \mathrm{OD}\right): \delta 8.25-8.17(\mathrm{~m}$, 2H), 8.12-8.04 (m, 2H), 8.03 (d, $J=0.9 \mathrm{~Hz}, 1 \mathrm{H}), 7.36$ (d, $J=0.9 \mathrm{~Hz}, 1 \mathrm{H}), 7.28-7.17$ (m, 4H), 5.14 (d, $J=5.5 \mathrm{~Hz}, 2 \mathrm{H}$ ), 4.33-4.22 (m, 1H), 2.94 (dd, $J=17.9,5.8 \mathrm{~Hz}, 1 \mathrm{H}), 2.78$ (dd, $J=17.9$, 7.0 Hz, 1H). HRMS (ESI+): m/z calcd for $\mathrm{C}_{20} \mathrm{H}_{18} \mathrm{~N}_{6} \mathrm{O}_{4}[\mathrm{M}+\mathrm{H}]^{+}$, 407.14623 found: 407.14612. 


\section{4-(Benzo[d]thiazol-2-yloxy)benzonitrile (S-29)}

Diarylether S-29 was prepared from 2-chlorobenzothiazole $(6.51 \mathrm{~mL}, 50.0 \mathrm{mmol})$ and 4-hydroxybenzonitrile $(6.55 \mathrm{~g}, 55.0 \mathrm{mmol})$ in analogy to S-26. Recrystallization from refluxing EtOAc/Heptane afforded title compound S-29 (6.51 g, 49\%) as a colorless solid. MS (ESI+/-): $\mathrm{m} / \mathrm{z}=+253.1 /-251.1 .{ }^{1} \mathrm{H}$ NMR (400 MHz, DMSO-d 6 ): $\delta$ 8.05-7.99 (m, 3H), 7.77-7.68 (m, 3H), 7.51-7.42 (m, 1H), 7.42-7.34 (m, 1H).

\section{2-(4-(2H-Tetrazol-5-yl)phenoxy)benzo[d]thiazole (S-30)}

Tetrazole S-30 was prepared in analogy to 27 from nitrile S-29 $(6.05 \mathrm{~g}, 24.0 \mathrm{mmol})$ and obtained as a beige powder (6.28 g, 87\%). MS (ESI+/-): m/z $=+296.1 /-294.1 .{ }^{1} \mathrm{H}$ NMR $(400 \mathrm{MHz}$, DMSO-d $\mathrm{d}_{6}$ ): $\delta 16.93$ (s, br, 1H), 8.20-8.12 (m, 2H), 8.01-7.94 (m, 1H), 7.75-7.67 (m, 3H), 7.44 (td, $J=7.7,1.4 \mathrm{~Hz}, 1 \mathrm{H}), 7.40-7.31(\mathrm{~m}, 1 \mathrm{H})$.

tert-butyl (S)-4-(5-(4-(benzo[d]thiazol-2-yloxy)phenyl)-2H-tetrazol-2-yl)-3-((tert-butoxycarbonyl)amino)butanoate [(S)-S-31]

Tetrazole S-30 (2.07 g, $7.00 \mathrm{mmol})$ was alkylated in analogy to $(\boldsymbol{R})$-S-11 to afford title compound (S)-S-31 after flash column chromatography on RP18 silica (0.1\% TFA in water:MeCN from 19:1 to $0: 1)$ as a yellow foam $(2.39 \mathrm{mg}, 62 \%)$ in ca. $90 \%$ purity. The material was carried forward without additional purification. MS (ESI + ): $\mathrm{m} / \mathrm{z}=+553.3$. ${ }^{1} \mathrm{H}$ NMR $\left(400 \mathrm{MHz}\right.$, DMSO- $\left.\mathrm{d}_{6}\right)$ : $\delta 8.20-8.14$ (m, 2H), 7.99 (d, $J=7.8 \mathrm{~Hz}, 1 \mathrm{H}), 7.73$ (d, $J=8.0 \mathrm{~Hz}, 1 \mathrm{H}), 7.71-7.64$ (m, 2H), 7.46 (td, $J=7.7,1.3 \mathrm{~Hz}, 1 \mathrm{H}$ ), 7.37 (t, $J=7.6 \mathrm{~Hz}, 1 \mathrm{H}$ ), 7.02 (d, $J=9.0 \mathrm{~Hz}, 1 \mathrm{H}), 4.86$ (dd, $J=13.6$, $4.2 \mathrm{~Hz}, 1 \mathrm{H}$ ), 4.65 (dd, $J=13.6,8.5 \mathrm{~Hz}, 1 \mathrm{H}$ ), 4.32 (tq, $J=9.1,4.5 \mathrm{~Hz}, 1 \mathrm{H}$ ), 2.64 (dd, $J=15.7,5.6$ Hz, 1H), 2.45 (dd, $J=15.6,9.0 \mathrm{~Hz}, 1 \mathrm{H}), 1.40$ (s, 9H), 1.26 (s, 9H).

(S)-3-Amino-4-(5-(4-(benzo[d]thiazol-2-yloxy)phenyl)-2H-tetrazol-2-yl)butanoic acid [(S)-20]

(S)-S-31 (2.39 g, 90\%, $3.89 \mathrm{mmol}$ ) was deprotected in analogy to $(\boldsymbol{S})$-22 to afford the hydrochloride of $(\boldsymbol{S})-20$ (1.14 g, 65\%) as a colorless powder. ${ }^{1} \mathrm{H}$ NMR (400 MHz, $\mathrm{CD}_{3} \mathrm{OD}$ ): $\delta$ 8.35-8.27 (m, 2H), 7.83 (dd, $J=8.1,1.2 \mathrm{~Hz}, 1 \mathrm{H}), 7.68$ (dd, $J=8.2,1.1 \mathrm{~Hz}, 1 \mathrm{H}$ ), 7.65-7.57 (m, 2H), 7.45 (td, $J=7.8,1.3 \mathrm{~Hz}, 1 \mathrm{H}$ ), 7.35 (td, $J=7.7,1.2 \mathrm{~Hz}, 1 \mathrm{H}$ ), 5.17 (d, $J=5.5 \mathrm{~Hz}, 2 \mathrm{H}$ ), $4.34-$ 
4.24 (m, 1H), 2.96 (dd, $J=17.9,5.8 \mathrm{~Hz}, 1 \mathrm{H}), 2.79$ (dd, $J=17.9,7.0 \mathrm{~Hz}, 1 \mathrm{H}$ ). HRMS (ESI+): m/z calcd for $\mathrm{C}_{18} \mathrm{H}_{16} \mathrm{~N}_{6} \mathrm{O}_{3} \mathrm{~S}[\mathrm{M}+\mathrm{H}]^{+}$, 397.10774 found: 397.10764.

tert-butyl (R)-4-(5-(4-(benzo[d]thiazol-2-yloxy)phenyl)-2H-tetrazol-2-yl)-3-((tert-butoxycarbonyl)amino)butanoate [(R)-S-31]

Tetrazole S-30 (2.07 g, $7.00 \mathrm{mmol}$ ) was alkylated in analogy to $(\boldsymbol{S})$-30 using reagent $(\boldsymbol{R})$-29 ( $2.12 \mathrm{~g}, 7.70 \mathrm{mmol})$. Flash column chromatography on RP18 silica (0.1\% TFA in water:MeCN from 19:1 to 0:1) afforded title compound (R)-S-31 (2.69 g, 66\%) as a dark yellow viscous oil. MS (ESI+/-): $\mathrm{m} / \mathrm{z}=+553.3 /-551.3 .{ }^{1} \mathrm{H}$ NMR (400 MHz, DMSO-d 6 ): $\delta$ 8.23-8.13 (m, 2H), 7.98 (dd, $J=8.0,1.3 \mathrm{~Hz}, 1 \mathrm{H}), 7.73$ (dd, $J=8.1,1.1 \mathrm{~Hz}, 1 \mathrm{H}$ ), 7.71-7.63 (m, 2H), 7.46 (td, $J=7.8$, $1.4 \mathrm{~Hz}, 1 \mathrm{H}$ ), 7.36 (td, $J=7.7,1.3 \mathrm{~Hz}, 1 \mathrm{H}), 7.02$ (d, $J=9.0 \mathrm{~Hz}, 1 \mathrm{H}), 4.86$ (dd, $J=13.6,4.3 \mathrm{~Hz}$, 1H), 4.65 (dd, $J=13.5,8.5 \mathrm{~Hz}, 1 \mathrm{H}$ ), 4.32 (tq, $J=9.0,4.4 \mathrm{~Hz}, 1 \mathrm{H}$ ), 2.64 (dd, $J=15.8,5.6 \mathrm{~Hz}, 1 \mathrm{H}$ ), 2.49-2.40 (m, 1H), 1.40 (s, 9H), 1.25 (s, 9H).

\section{(R)-3-Amino-4-(5-(4-(benzo[d]thiazol-2-yloxy)phenyl)-2H-tetrazol-2-yl)butanoic acid} [(R)-20]

(R)-S-31 (2.69 g, $4.87 \mathrm{mmol}$ ) was deprotected in analogy to (S)-22 to afford the hydrochloride of (R)-20 (1.41 g, 63\%) as a colorless powder. ${ }^{1} \mathrm{H}$ NMR (400 MHz, $\left.\mathrm{CD}_{3} \mathrm{OD}\right): \delta 8.36-8.28$ (m, 2H), 7.85 (d, $J=8.0 \mathrm{~Hz}, 1 \mathrm{H}), 7.70$ (d, $J=8.1 \mathrm{~Hz}, 1 \mathrm{H}), 7.65-7.58$ (m, 2H), 7.46 (t, $J=7.7 \mathrm{~Hz}, 1 \mathrm{H}), 7.36$ (t, $J=7.7 \mathrm{~Hz}, 1 \mathrm{H}$ ), 5.19-5.05 (m, 2H), 4.23-4.15 (m, 1H), 2.80 (dd, $J=17.4,5.1 \mathrm{~Hz}, 1 \mathrm{H}$ ), 2.62 (dd, $J=17.4,8.0 \mathrm{~Hz}, 1 \mathrm{H}$ ). HRMS (ESI+): $\mathrm{m} / \mathrm{z}$ calcd for $\mathrm{C}_{18} \mathrm{H}_{16} \mathrm{~N}_{6} \mathrm{O}_{3} \mathrm{~S}[\mathrm{M}+\mathrm{H}]^{+}$, 397.10774 found: 397.10773.

\section{4-((5-Chloropyridin-2-yl)oxy)benzonitrile (S-32)}

A suspension of 4-hydroxybenzonitrile (3.00 g, $25.2 \mathrm{mmol}$ ), 2,5-dichloropyridine (3.91 g, $26.4 \mathrm{mmol}$ ) and solid $\mathrm{K}_{2} \mathrm{CO}_{3}$ in DMF (8 mL) was heated to $140^{\circ} \mathrm{C}$ for 2 days. The $\mathrm{RM}$ was diluted with EtOAc:Heptane (1:1, $150 \mathrm{~mL})$, washed with water $(2 \times 100 \mathrm{~mL})$, sat. $\mathrm{NaHCO}_{3}(100 \mathrm{~mL})$ and brine ( $50 \mathrm{~mL}$ ), dried over $\mathrm{Na}_{2} \mathrm{SO}_{4}$, filtered and concentrated $i$. vac.. The crude was triturated with $\mathrm{MeOH}(100 \mathrm{~mL})$, filtered, washed with $\mathrm{MeOH}(2 \times 10 \mathrm{~mL})$, and dried to afford title compound S-32 (2.30 g, 39\%) as a colorless powder. MS (ESI+): $\mathrm{m} / \mathrm{z}=+230.9 .{ }^{1} \mathrm{H}$ NMR $(400 \mathrm{MHz}$, 
DMSO-d $\mathrm{d}_{6}$ ): $\delta 8.26$ (d, $\left.J=2.7 \mathrm{~Hz}, 1 \mathrm{H}\right), 8.04$ (dd, $\left.J=8.7,2.8 \mathrm{~Hz}, 1 \mathrm{H}\right), 7.95-7.86$ (m, 2H), 7.40$7.31(\mathrm{~m}, 2 \mathrm{H}), 7.24(\mathrm{~d}, J=8.7 \mathrm{~Hz}, 1 \mathrm{H})$.

\section{2-(4-(2H-Tetrazol-5-yl)phenoxy)-5-chloropyridine (S-33)}

Tetrazole S-33 was prepared in analogy to 27 from nitrile S-32 (1.50 g, $6.50 \mathrm{mmol})$ and obtained as a colorless powder (1.42 g, 77\%). MS (ESI+/-): m/z $=+274.0 /-272.0 .{ }^{1} \mathrm{H}$ NMR $(400 \mathrm{MHz}$, DMSO-d $\mathrm{d}_{6}$ ): $\delta 16.80$ (s, br, 1H), 8.26 (d, $J=2.7 \mathrm{~Hz}, 1 \mathrm{H}$ ), 8.12-8.06 (m, 2H), 8.03 (dd, $J=8.8$, $2.7 \mathrm{~Hz}, 1 \mathrm{H}), 7.43-7.36$ (m, 2H), 7.22 (d, $J=8.8 \mathrm{~Hz}, 1 \mathrm{H})$.

tert-Butyl (R)-3-((tert-butoxycarbonyl)amino)-4-(5-(4-((5-chloropyridin-2-yl)oxy)phenyl)2H-tetrazol-2-yl)butanoate [(R)-S-34]

Tetrazole S-33 (300 mg, $1.10 \mathrm{mmol}$ ) was alkylated in analogy to $(\boldsymbol{R})$-S-11. Flash column chromatography on RP18 silica (0.1\% TFA in water:MeCN from 19:1 to 0:1) afforded title compound (R)-S-34 (463 mg, 59\%) as a colorless solid in ca. 90\% purity. MS (ESI+/-): $\mathrm{m} / \mathrm{z}=+534.2 /-531.1$. The material was carried forward without additional purification.

(R)-3-Amino-4-(5-(4-((5-chloropyridin-2-yl)oxy)phenyl)-2H-tetrazol-2-yl)butanoic acid [(R)-21]

(R)-S-34 (463 g, $0.872 \mathrm{mmol}$ ) was deprotected in analogy to (S)-22 to afford the hydrochloride of (R)-21 (322 mg, 80\%) as a colorless powder. ${ }^{1} \mathrm{H}$ NMR (400 MHz, DMSO-d 6 ): $\delta 12.94$ (s, br, $1 \mathrm{H}$ ), 8.50-8.37 (m, 3H), 8.26 (d, $J=2.7 \mathrm{~Hz}, 1 \mathrm{H}), 8.17-8.09$ (m, 2H), 8.03 (dd, $J=8.7,2.7 \mathrm{~Hz}, 1 \mathrm{H}$ ), 7.40-7.32 (m, 2H), 7.21 (d, $J=8.8 \mathrm{~Hz}, 1 \mathrm{H}$ ), 5.14-5.06 (m, 2H), 4.08 (q, $J=5.8 \mathrm{~Hz}, 1 \mathrm{H}$ ), 2.81 (d, $J=6.5 \mathrm{~Hz}, 2 \mathrm{H})$. HRMS (ESI+): $\mathrm{m} / \mathrm{z}$ calcd for $\mathrm{C}_{16} \mathrm{H}_{15} \mathrm{ClN}_{6} \mathrm{O}_{3}[\mathrm{M}+\mathrm{H}]^{+}, 375.09669$ found: 375.09668 .

tert-Butyl (R)-3-((tert-butoxycarbonyl)amino)-4-(5-(4-((5-chloro-3-fluoropyridin-2yl)oxy)phenyl)-2H-tetrazol-2-yl)butanoate [(R)-30]

Tetrazole 27 (1.00 g, $3.15 \mathrm{mmol}$ ) was alkylated in analogy to $(\boldsymbol{S})$-30 using reagent $(\boldsymbol{R})-29$ (1.28 g, 3.79 mmol). Flash column chromatography on RP18 silica (0.1\% TFA in water:MeCN from 19:1 
to 0:1) afforded title compound $(\boldsymbol{R})-30(1.56 \mathrm{~g}, 88 \%)$ as a viscous yellow oil. MS (ESI+/-): $\mathrm{m} / \mathrm{z}=+549.2 /-547.3$.

(R)-3-Amino-4-(5-(4-((5-chloro-3-fluoropyridin-2-yl)oxy)phenyl)-2H-tetrazol-2-yl)butanoic acid $[(R)-22]$

(R)-30 (1.56 g, $2.84 \mathrm{mmol}$ ) was deprotected in analogy to $(S)$-22 to afford the hydrochloride of (R)-22 (1.14 g, 92\%) as a colorless powder. ${ }^{1} \mathrm{H}$ NMR (400 MHz, CD $\left.{ }_{3} \mathrm{OD}\right): \delta 8.28-8.20(\mathrm{~m}, 2 \mathrm{H})$, 7.98 (d, $J=2.2 \mathrm{~Hz}, 1 \mathrm{H}$ ), 7.92 (dd, $J=9.5,2.2 \mathrm{~Hz}, 1 \mathrm{H}$ ), 7.40-7.32 (m, 2H), 5.17 (d, $J=5.5 \mathrm{~Hz}$, 2H), 4.30 (dq, $J=7.0,5.6 \mathrm{~Hz}, 1 \mathrm{H}$ ), 2.96 (dd, $J=17.9,5.8 \mathrm{~Hz}, 1 \mathrm{H}$ ), 2.80 (dd, $J=17.9,7.0 \mathrm{~Hz}$, 1H). ${ }^{19} \mathrm{~F}$ NMR (376 MHz, DMSO-d 6 ): $\delta-133.95$ (d, $J=11.3 \mathrm{~Hz}, 1 \mathrm{~F}$ ). HRMS (ESI+): $\mathrm{m} / \mathrm{z}$ calcd for $\mathrm{C}_{16} \mathrm{H}_{14} \mathrm{ClFN}_{6} \mathrm{O}_{3}[\mathrm{M}+\mathrm{H}]^{+}$, 393.08727 found: 393.08731 .

\section{5-(4-((tert-Butyldimethylsilyl)oxy)phenyl)-2H-tetrazole (S-35)}

Tetrazole S-35 was prepared in analogy to 27 from 4-((tert-butyldimethylsilyl)oxy)benzonitrile $(8.00 \mathrm{~g}, 34.3 \mathrm{mmol})$ and obtained as a colorless powder $(8.9 \mathrm{~g}, 92 \%)$. MS (ESI+/-): $\mathrm{m} / \mathrm{z}=$ +277.1/-275.1. ${ }^{1} \mathrm{H}$ NMR (400 MHz, $\mathrm{CDCl}_{3}$ ): $\delta 8.05-7.93$ (m, 2H), 7.05-6.93 (m, 2H), 1.04-0.95 (m, 9H), 0.27-0.21 (m, $6 \mathrm{H})$.

tert-Butyl (R)-3-((tert-butoxycarbonyl)amino)-4-(5-(4-((tert-butyldimethylsilyl)oxy)phenyl)$2 H$-tetrazol-2-yl)butanoate [(R)-S-36]

Tetrazole S-35 (4.00 g, $14.5 \mathrm{mmol}$ ) was alkylated in analogy to $(\boldsymbol{R})$-S-11. Purification by flash column chromatography on RP18 silica (0.1\% TFA in water:MeCN from 19:1 to 0:1) enriched the purity of the intermediate $(\boldsymbol{R})-\mathrm{S}-36$ to ca. $80 \%$ (4.50 g, 36\%). MS (ESI+): $\mathrm{m} / \mathrm{z}=+534.4$. Used directly in the next step.

tert-Butyl (R)-3-((tert-butoxycarbonyl)amino)-4-(5-(4-hydroxyphenyl)-2H-tetrazol-2yl)butanoate [(R)-S-37]

A solution of $(\boldsymbol{R})-\mathrm{S}-36(4.50 \mathrm{~g}, 80 \%, 6.74 \mathrm{mmol})$ in THF $(16.9 \mathrm{~mL})$ was cooled to $0^{\circ} \mathrm{C}$, treated with TBAF (1M in THF, $7.4 \mathrm{~mL}$ ) and stirred for $2 \mathrm{~h}$. The RM was concentrated i. vac., adsorbed on Isolute $^{\mathrm{TM}}$ and purified by flash column chromatography on RP18 silica $(0.1 \%$ TFA in 
water:MeCN from 19:1 to 0:1) to afford title compound ( $R$ )-S-37 (2.76 g, 74\%) as a colorless powder. MS (ESI+/-): m/z = +420.3/-418.3. ${ }^{1} \mathrm{H}$ NMR (400 MHz, CD $\left.{ }_{3} \mathrm{OD}\right): \delta$ 7.97-7.89 (m, 2H), 6.93-6.85 (m, 2H), 4.83 (d, $J=4.6 \mathrm{~Hz}, 1 \mathrm{H}$ ), 4.71 (dd, $J=13.6,8.0 \mathrm{~Hz}, 1 \mathrm{H}$ ), 4.46 (tdd, $J=8.4$, 5.8, $4.5 \mathrm{~Hz}, 1 \mathrm{H}$ ), 2.62 (dd, $J=15.8,5.9 \mathrm{~Hz}, 1 \mathrm{H}$ ), 2.50 (dd, $J=15.8,8.4 \mathrm{~Hz}, 1 \mathrm{H}$ ), 1.46 (s, 9H), 1.32 (s, 9H).

tert-Butyl (R)-3-((tert-butoxycarbonyl)amino)-4-(5-(4-((5-methoxypyrimidin-2yl)oxy)phenyl)-2H-tetrazol-2-yl)butanoate [(R)-S-38]

A suspension of $(\boldsymbol{R})-\mathrm{S}-37(100 \mathrm{mg}, 0.238 \mathrm{mmol})$, 2-chloro-5-methoxypyrimidine (103 $\mathrm{mg}$, $0.715 \mathrm{mmol}$ ) and $\mathrm{Cs}_{2} \mathrm{CO}_{3}(155 \mathrm{mg}, 0.477 \mathrm{mmol})$ in $\mathrm{DMF}(0.8 \mathrm{~mL})$ was heated to $90^{\circ} \mathrm{C}$ for $3 \mathrm{~h}$. The RM was adsorbed on Isolute ${ }^{\mathrm{TM}}$ and purified by flash column chromatography on RP18 silica (0.1\% TFA in water:MeCN from 19:1 to 0:1) to afford title compound (R)-S-38 (92 $\mathrm{mg}, 72 \%$ ) as a colorless powder. MS (ESI+): $\mathrm{m} / \mathrm{z}=+528.5 .{ }^{1} \mathrm{H}$ NMR $\left(400 \mathrm{MHz}, \mathrm{DMSO}-\mathrm{d}_{6}\right): \delta 8.48-8.42(\mathrm{~m}$, 2H), 8.13-8.05 (m, 2H), 7.37-7.31 (m, 2H), 7.03-6.99 (m, 1H), 4.83 (dd, $J=13.8,4.3 \mathrm{~Hz}, 1 \mathrm{H}$ ), 4.63 (dd, $J=13.6,8.4 \mathrm{~Hz}, 1 \mathrm{H}$ ), 4.32 (td, $J=9.6,5.1 \mathrm{~Hz}, 1 \mathrm{H}), 3.92-3.86$ (m, 3H), 2.66-2.55 (m, 1H), 2.50-2.38 (m, 1H), 1.40 (s, 9H), 1.27 (s, 9H).

\section{(R)-3-Amino-4-(5-(4-((5-methoxypyrimidin-2-yl)oxy)phenyl)-2H-tetrazol-2-yl)butanoic acid $[(R)-23]$}

(R)-S-38 (78.0 $\mathrm{mg}, 0.127 \mathrm{mmol}$ ) was deprotected in analogy to $(\boldsymbol{S})$-22 to afford the hydrochloride of $(\boldsymbol{R})$-23 (33.0 mg, 61\%) as a colorless powder. ${ }^{1} \mathrm{H}$ NMR (400 MHz, $\mathrm{CD}_{3} \mathrm{OD}$ ): $\delta 8.38$ (s, 2H), 8.27-8.19 (m, 2H), 7.38-7.31 (m, 2H), 5.17 (d, $J=5.4$ Hz, 2H), 4.35-4.25 (m, 1H), 3.95 (s, 3H), 2.97 (dd, $J=17.9,5.8 \mathrm{~Hz}, 1 \mathrm{H}$ ), 2.81 (dd, $J=17.9,6.9 \mathrm{~Hz}, 1 \mathrm{H}$ ). HRMS (ESI+): m/z calcd for $\mathrm{C}_{16} \mathrm{H}_{17} \mathrm{~N}_{7} \mathrm{O}_{4}[\mathrm{M}+\mathrm{H}]^{+}, 372.14180$ found: 372.14160 . 
Instrument [BS_SQ17] WSJ-507.1.07

3: UV Detector: TAC: Wavelength Range: (210 - 450)

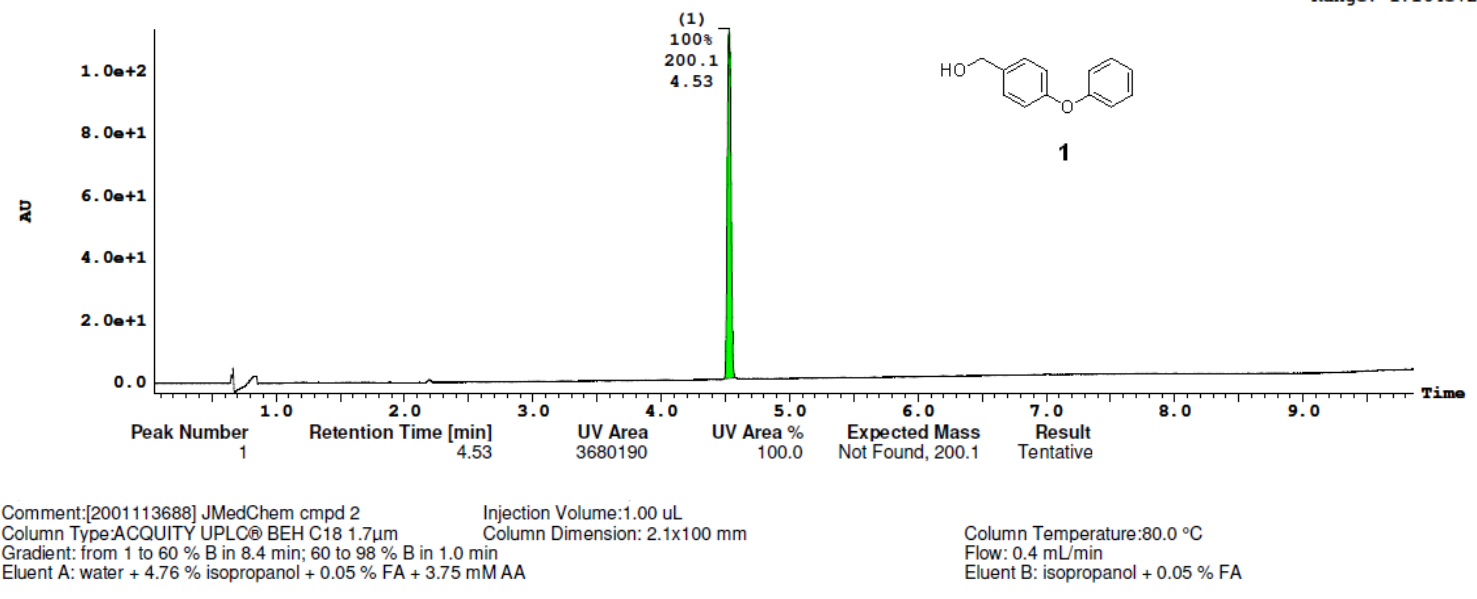

Instrument [BS_SQ18] WSJ-507.1.07

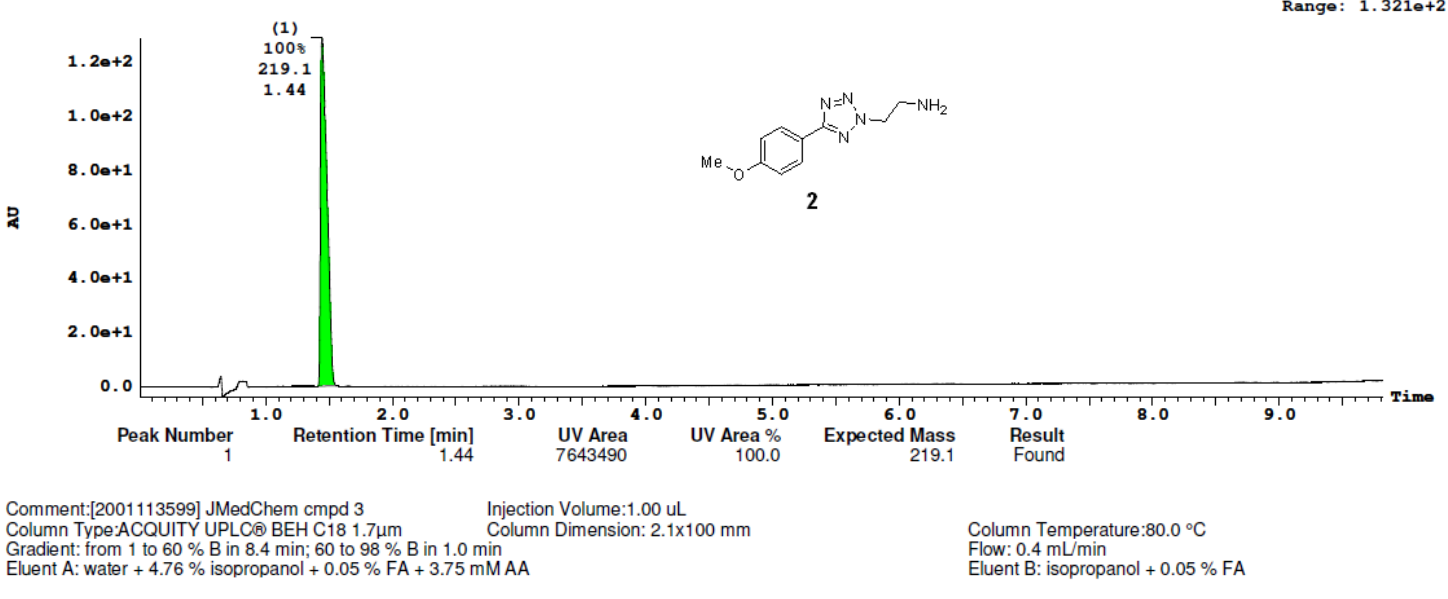

Instrument [BS_SQ03] WSJ-507.1.07

3: UV Detector: TAC: Wavelength Range: $(210-450)$

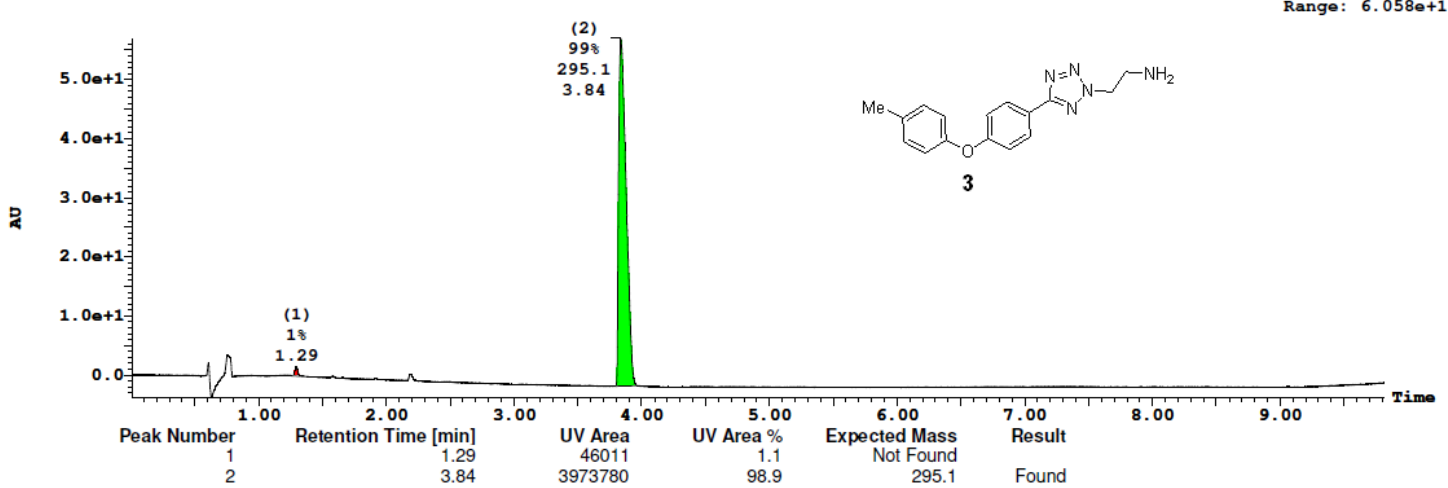


Comment:[2001113913] JMedChem cmpd $4 \quad$ Injection Volume:1.00 uL

Column Type:ACQUITY UPLC® BEH C18 1.7um Column Dimension: $2.1 \times 100 \mathrm{~mm} \quad$ Column Temperature:80.0 $00^{\circ} \mathrm{C}$

Gradient: from 1 to $60 \%$ B in 8.4 min; $601098 \%$ in 1.0 min

Instrument [BS_SQ16] WSJ-507.1.07

3: UV Detector: TAC: Wavelength Range: $(210-450)$

$904 e+1$

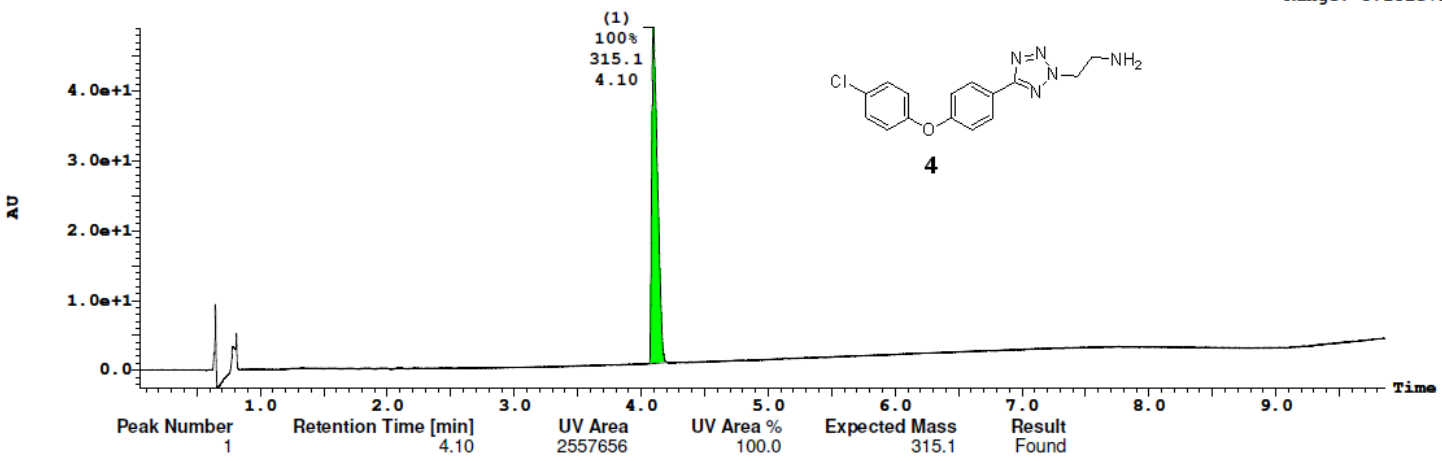

Comment:[2001112707] JMedChem cmpd 5 Injection Volume:1.00 uL

Column Type:ACQUITY UPLCB BEH C18 1.7um Column Dimension: 2.1x100 mm Column Temperature:80.0

Gradient: rom 1 to $60 \%$ B in 8.4 min; $6001098 \%$ in $1.0 \mathrm{~min}$ M

Eluent B: isopropanol $+0.05 \% \mathrm{FA}$

Instrument [BS_SQ17] WSJ-507.1.07

3: UV Detector: TAC: Wavelength Range: $(210-450)$

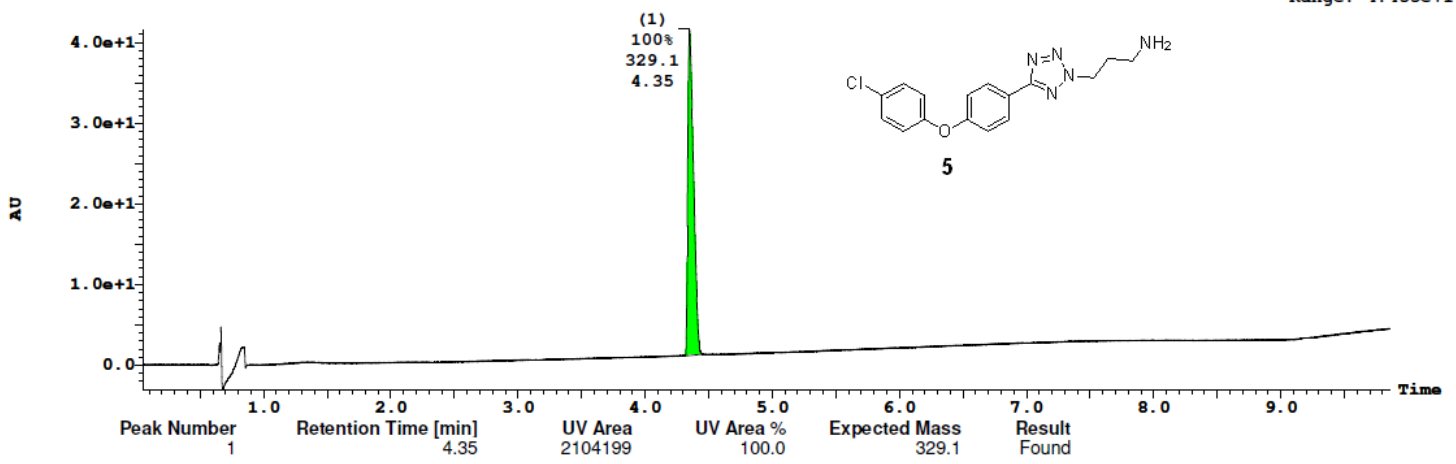

Comment:[2001114425] JMedChem cmpd $6 \quad$ Injection Volume:1.00 uL

Column Type:ACQUITY UPLCQ BEH C18 $1.7 \mu \mathrm{m} \quad$ Column Dimension: 2.1x100 mm Column Temperature:80.0 $0^{\circ} \mathrm{C}$

Eluent A: water $+4.76 \%$ isopropanol $+0.05 \% \mathrm{FA}+3.75 \mathrm{mMAA}$

Eluent B: isopropanol $+0.05 \% \mathrm{FA}$

Instrument [BS_SQ18] WSJ-507.1.07

3: UV Detector: TAC: Wavelength Range: $(210-450)$

Range: $5.8920+1$

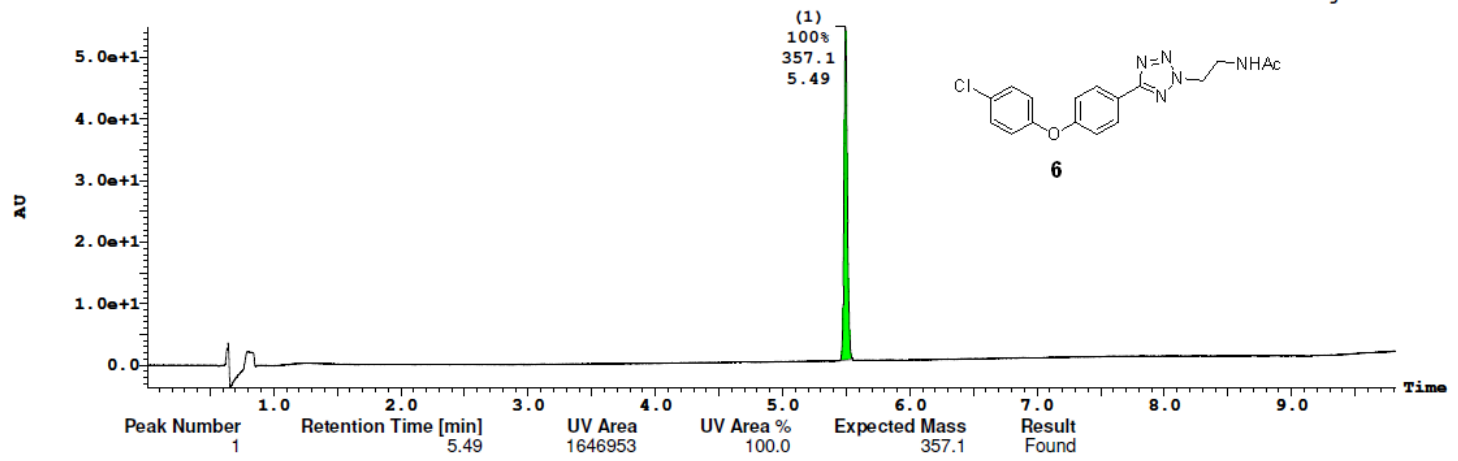


Instrument [BS_SQ16] WSJ-507.1.07

3: UV Detector: TAC: Wavelength Range: $(210-450)$

$6.8180+$

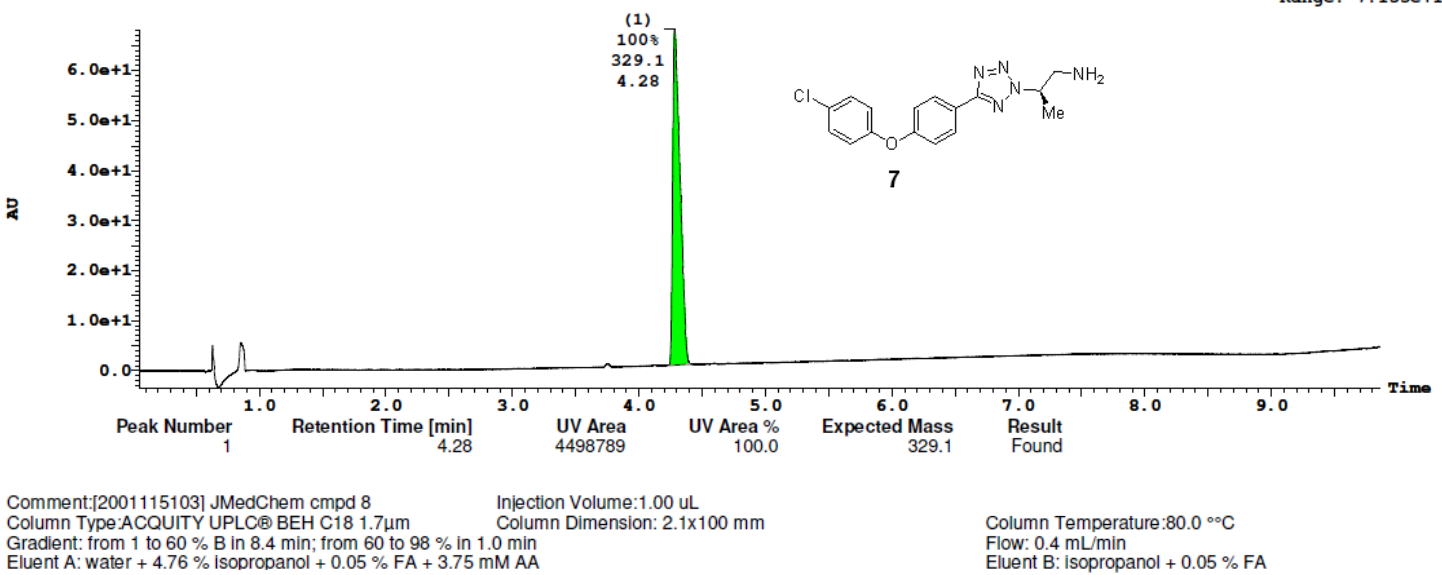

Instrument [BS_SQ09] WSJ-507.1.07

3: UV Detector: TAC: Wavelength Range: $(210-450)$

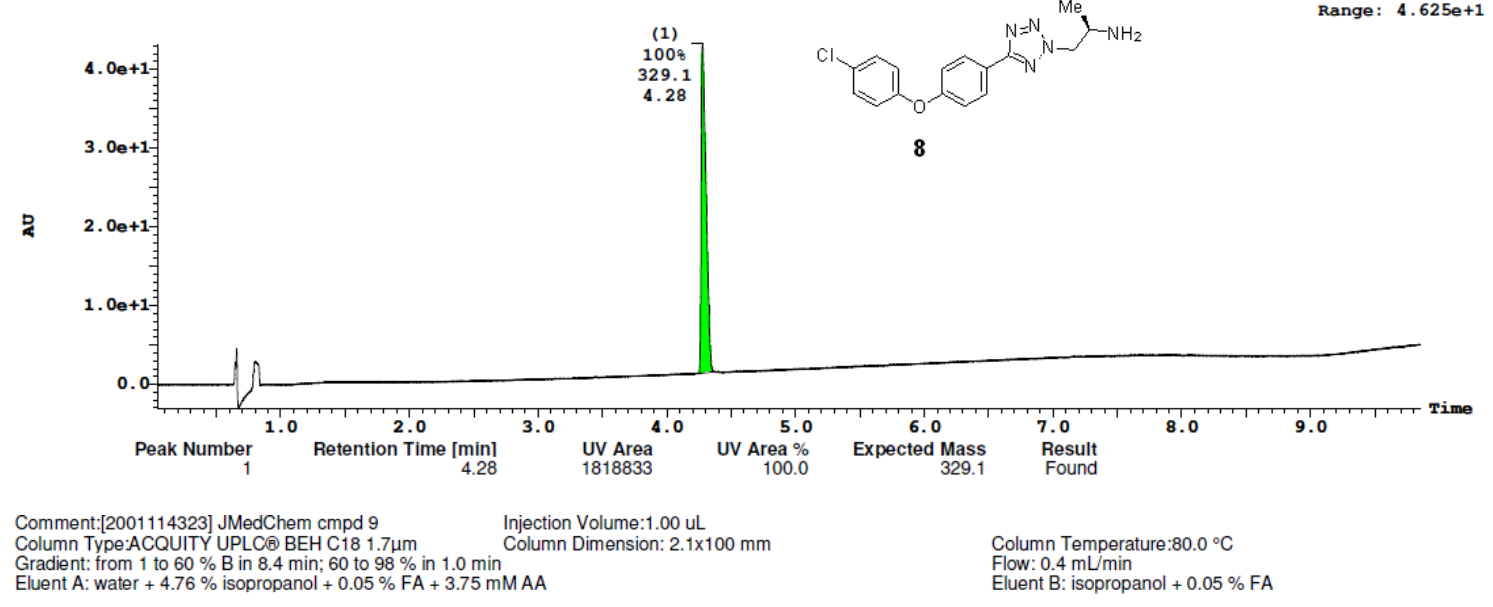

Instrument [BS_SQ16] WSJ-507.1.07

3: UV Detector: TAC: Wavelength Range: (210 - 450)

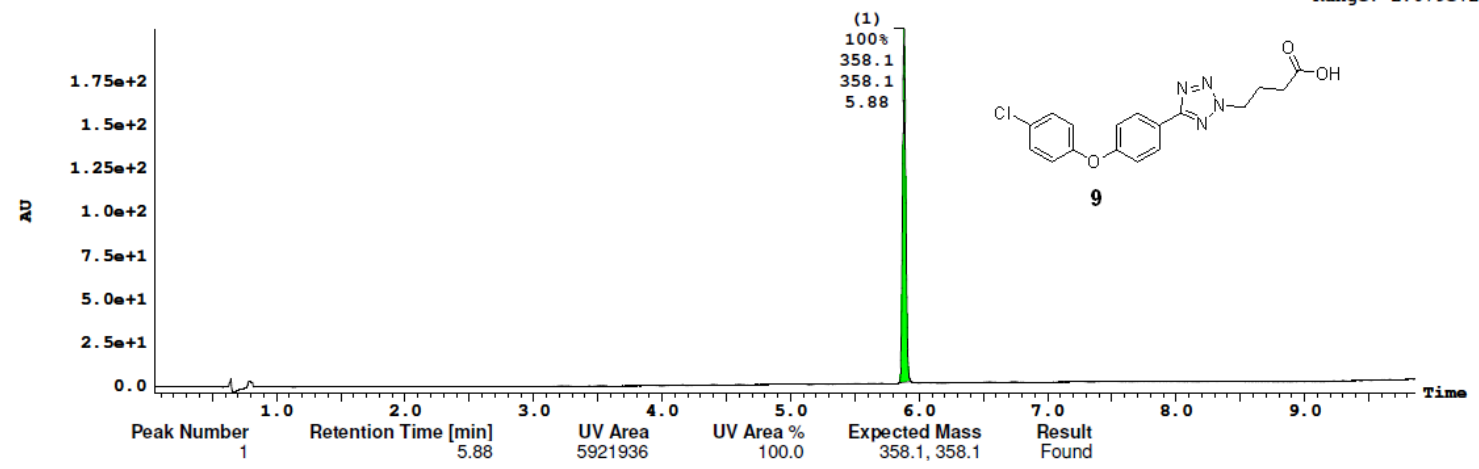


Instrument [BS_SQ17] WSJ-507.1.07

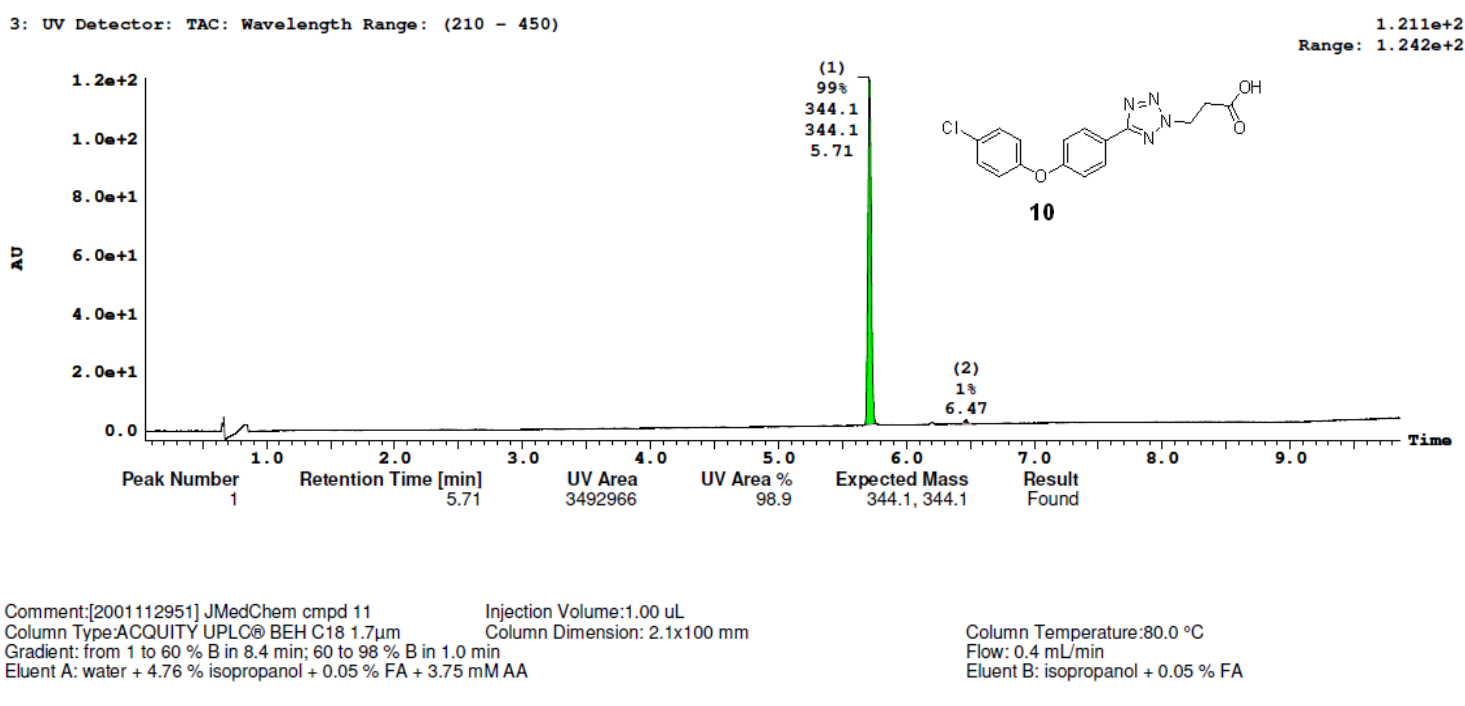

Instrument [BS_SQ11] WSJ-507.1.07

3: UV Detector: TAC: Wavelength Range: $(210-459)$

$6.232 e+1$

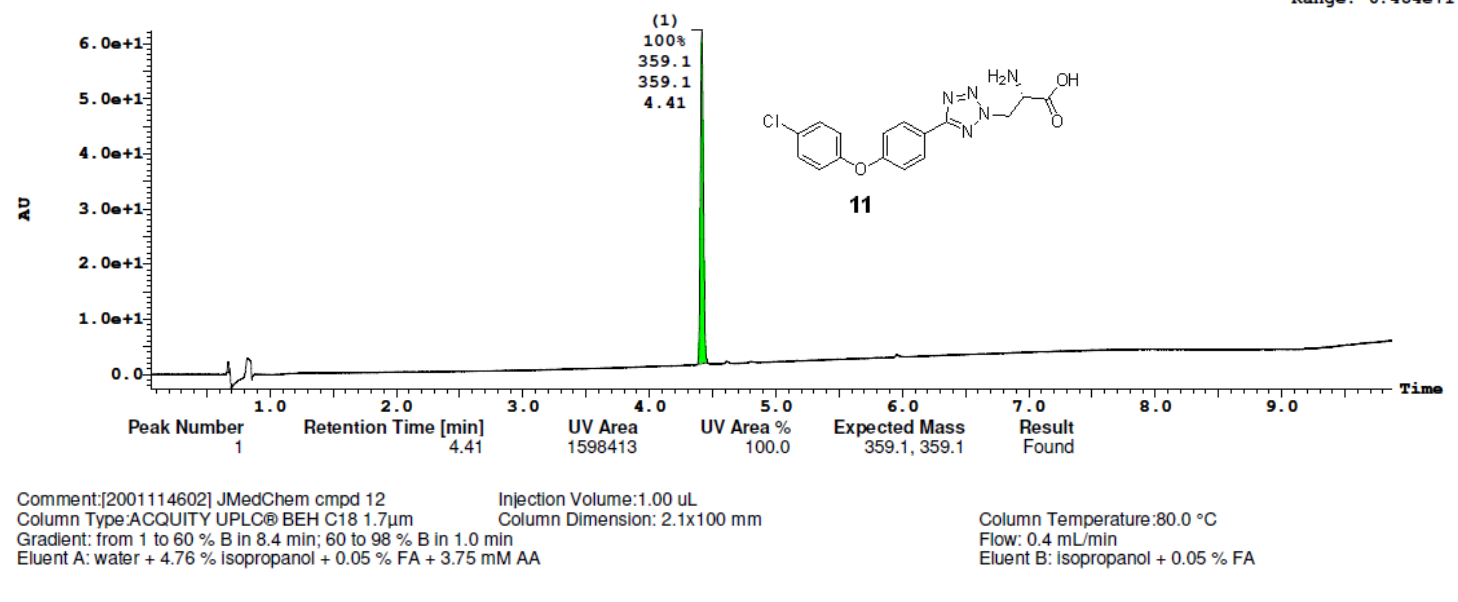

Instrument [BS_SQ11] WSJ-507.1.07

3: UV Detector: TAC: wavelength Range: $(210-459)$

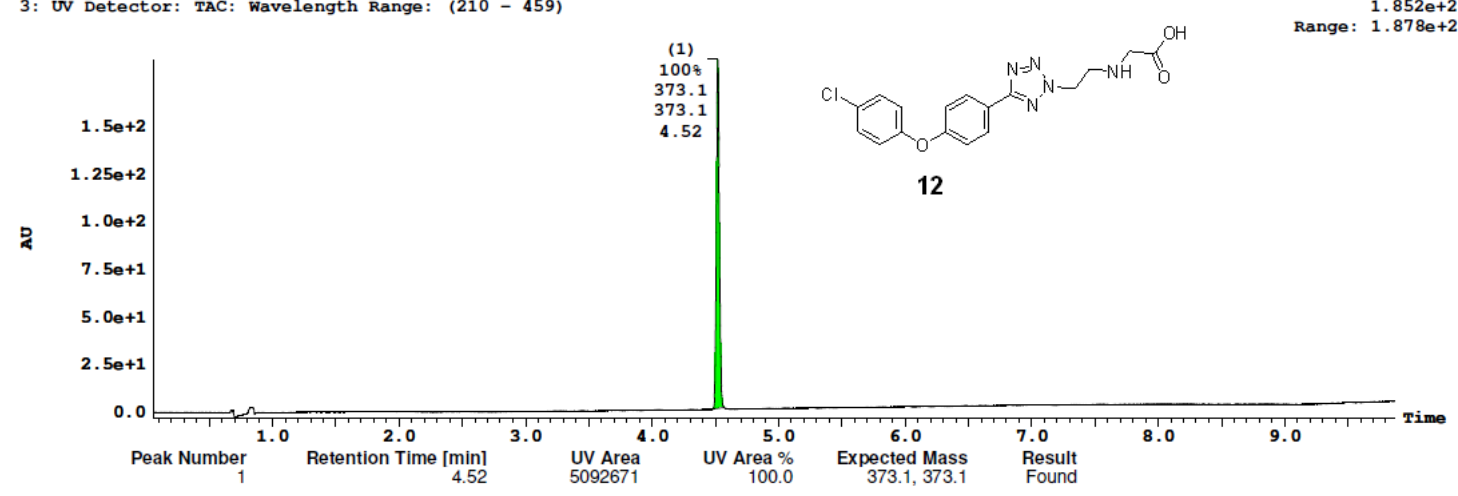


Instrument [BS_SQ03] WSJ-507.1.07

3: UV Detector: TAC: Wavelength Range: $(210-450)$

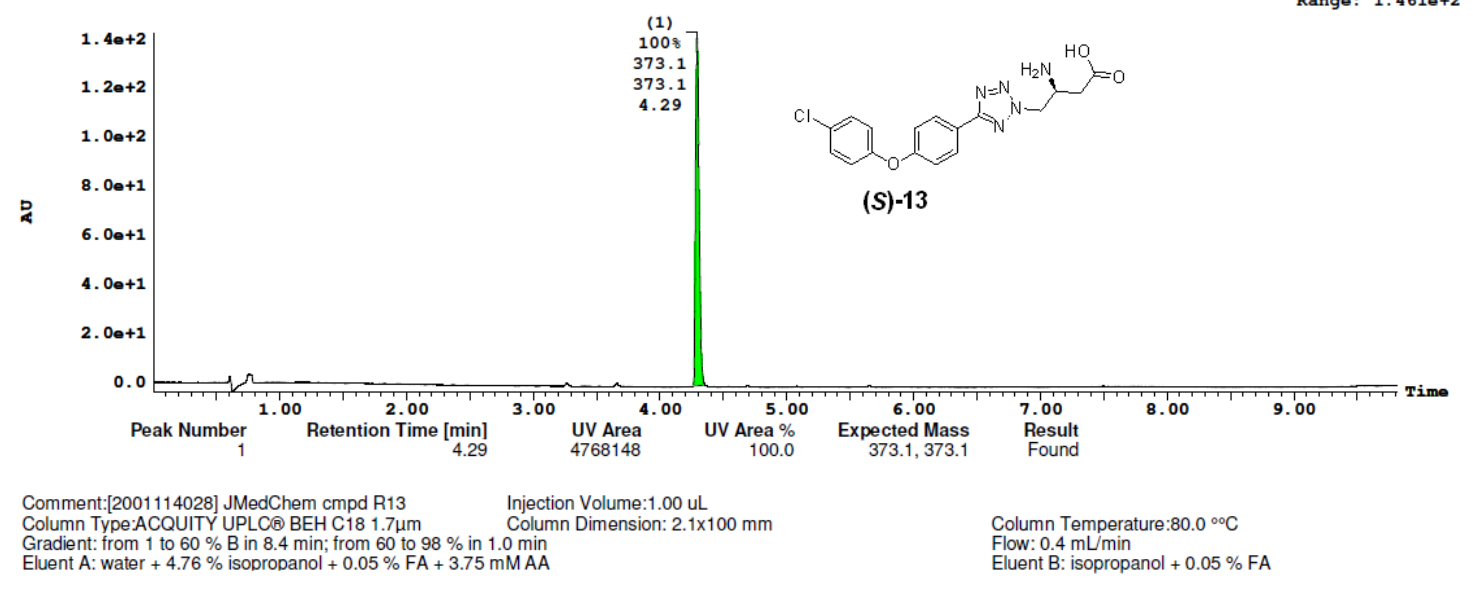

Instrument [BS_SQ09] WSJ-507.1.07

3: UV Detector: TAC: Wavelength Range: $(210-450)$

$6.513 e+1$

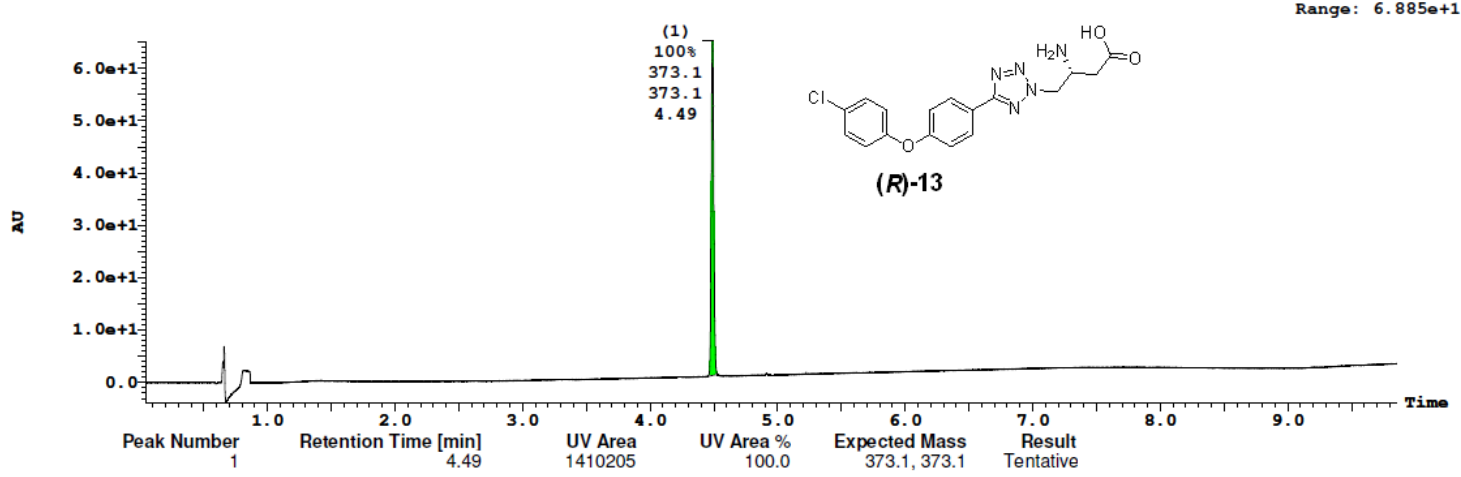

Instrument [BS_SQ03] WSJ-507.1.07

3: UV Detector: TAC: Wavelength Range: $(210-450)$

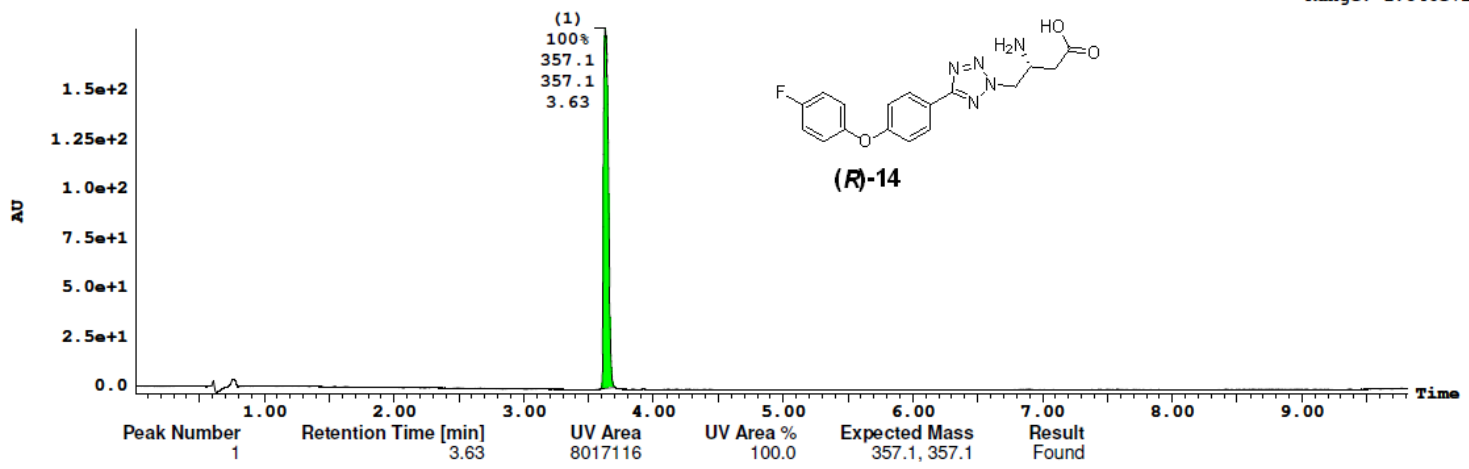


Instrument [BS_SQ11] WSJ-507.1.07

3: UV Detector: TAC: Wavelength Range: $(210-459)$

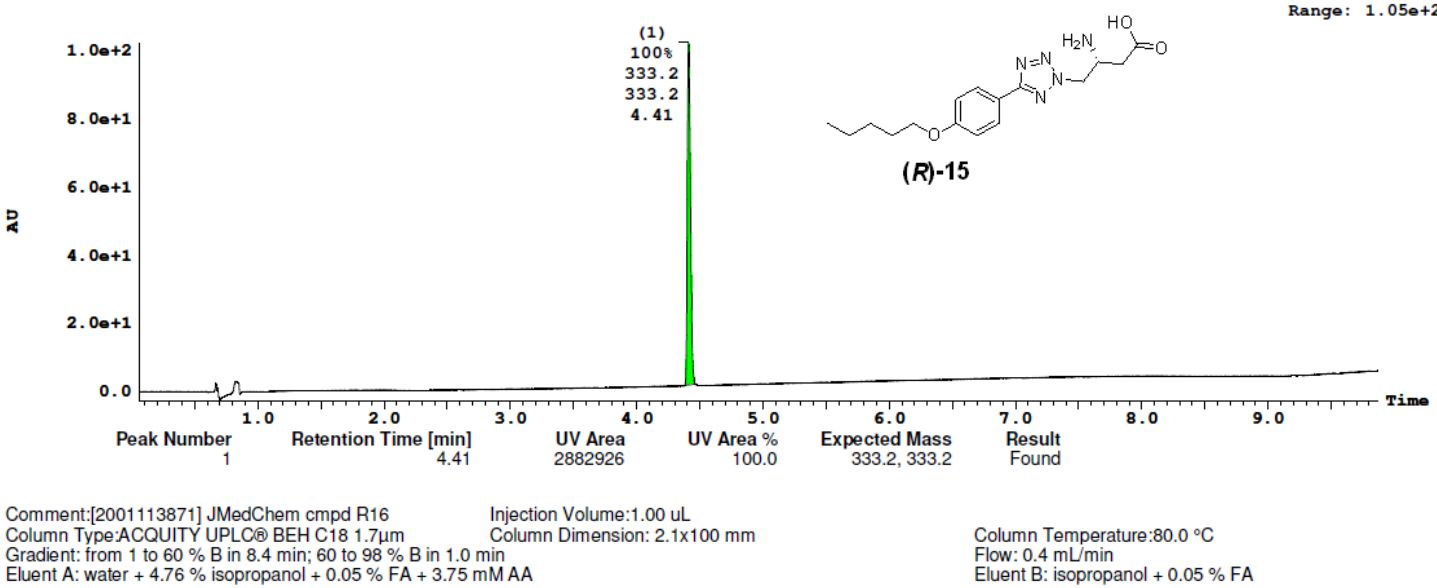

Instrument [BS_SQ18] WSJ-507.1.07

3: UV Detector: TAC: Wavelength Range: $(210-450)$

$1.581 e+2$

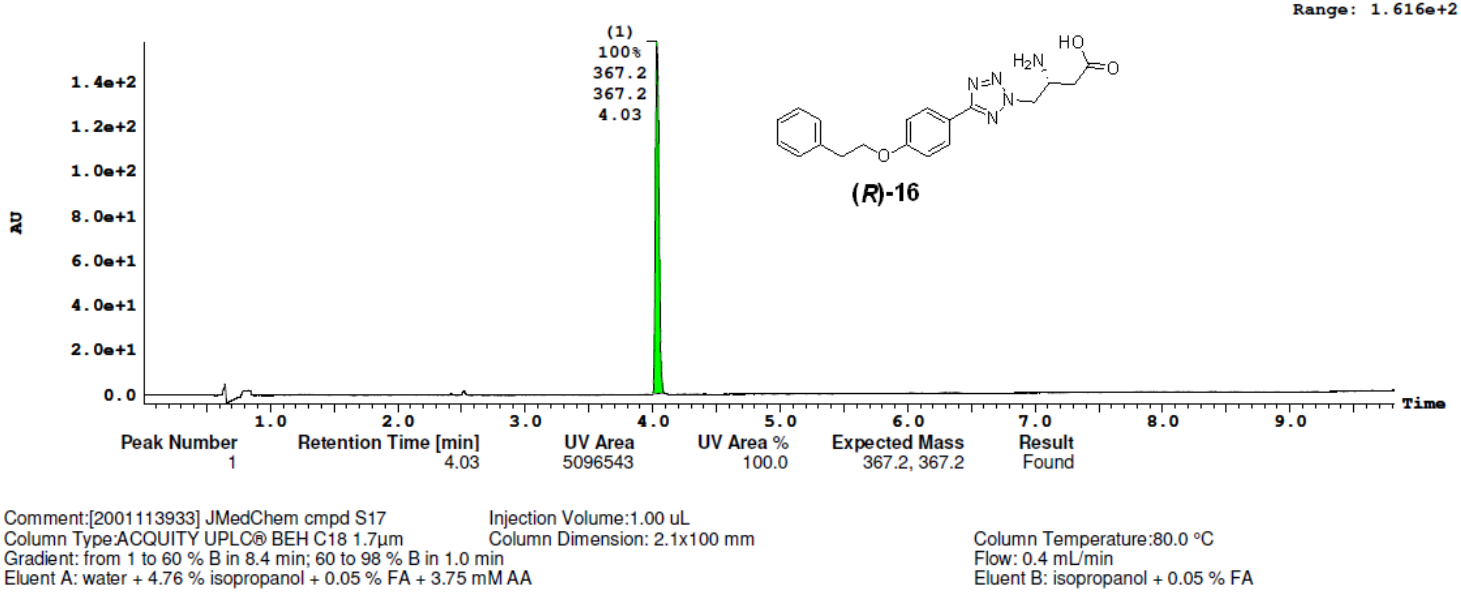

Instrument [BS_SQ03] WSJ-507.1.07

3: UV Detector: TAC: Wavelength Range: $(210-450)$

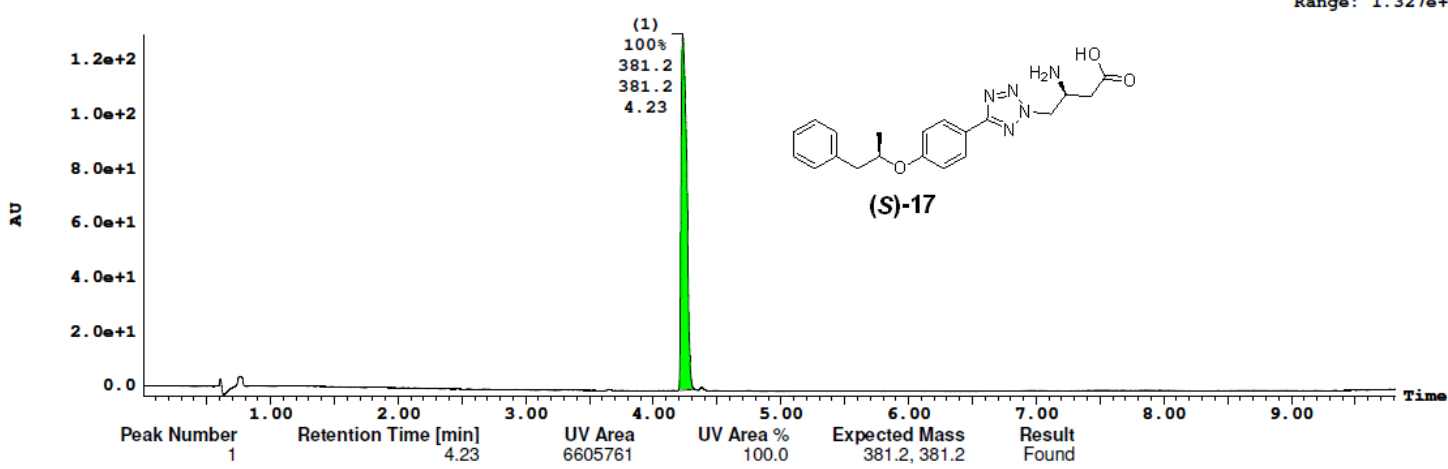


Instrument [BS_SQ09] WSJ-507.1.07

3: UV Detector: TAC: Wavelength Range: $(210-450)$

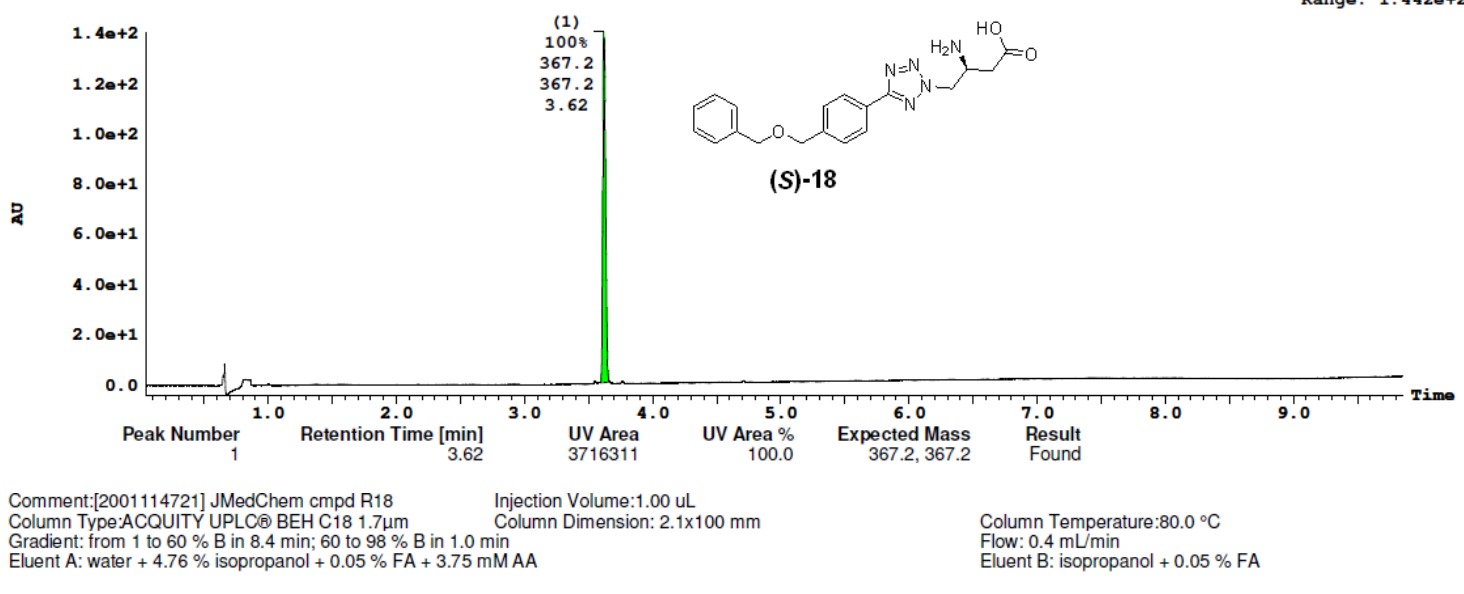

Instrument [BS_SQ18] WSJ-507.1.07

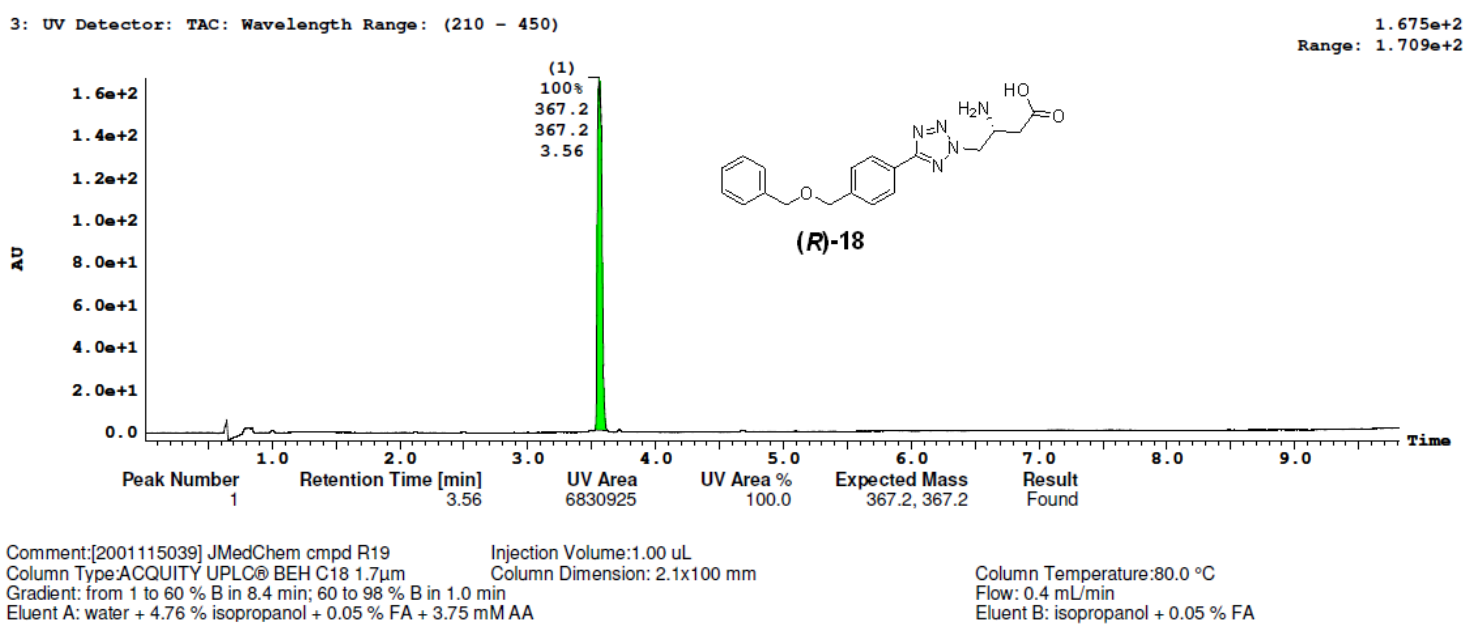

Instrument [BS_SQ11] WSJ-507.1.07

3: UV Detector: TAC: Wavelength Range: (210 - 459)

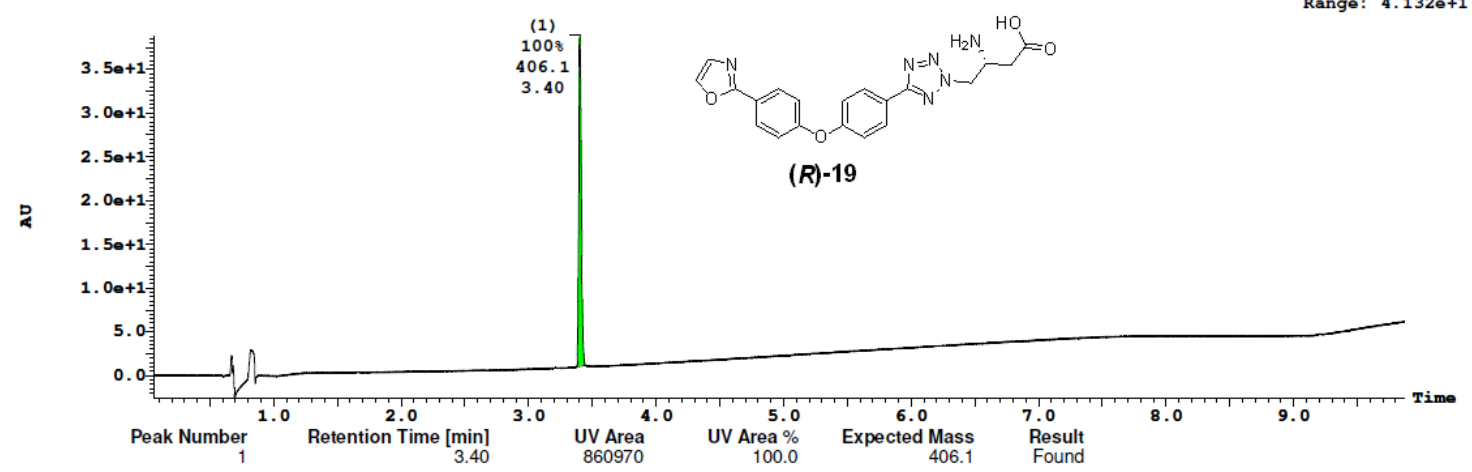


Instrument [BS_SQ17] WSJ-507.1.07

3: UV Detector: TAC: Wavelength Range: $(210-450)$

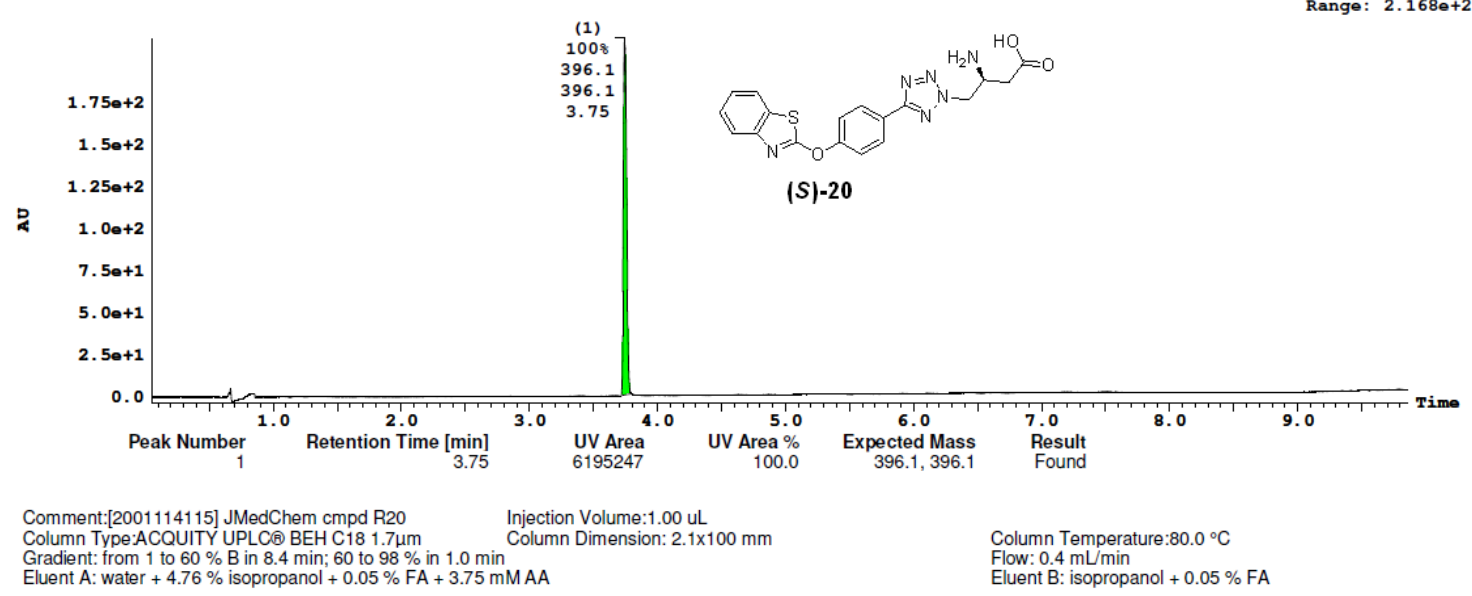

Instrument [BS_SQ17] WSJ-507.1.07

3: UV Detector: TAC: Wavelength Range: $(210-450)$

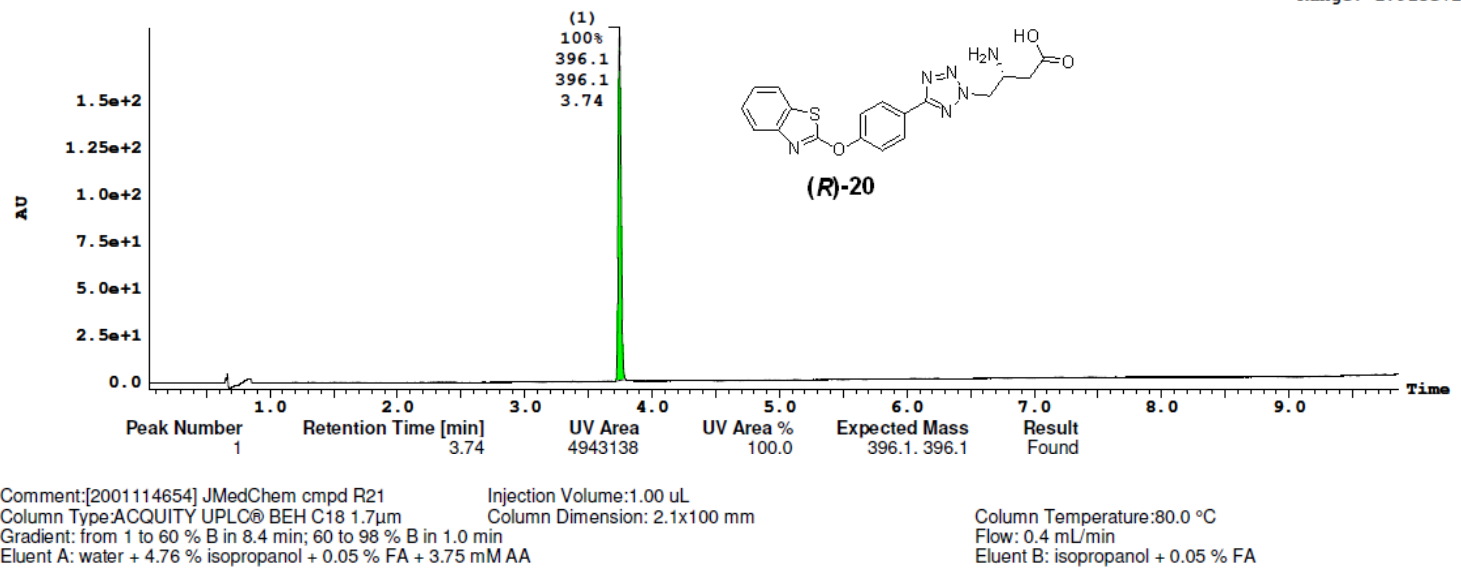

Instrument [BS_SQ11] WSJ-507.1.07

3: UV Detector: TAC: Wavelength Range: $(210-459)$

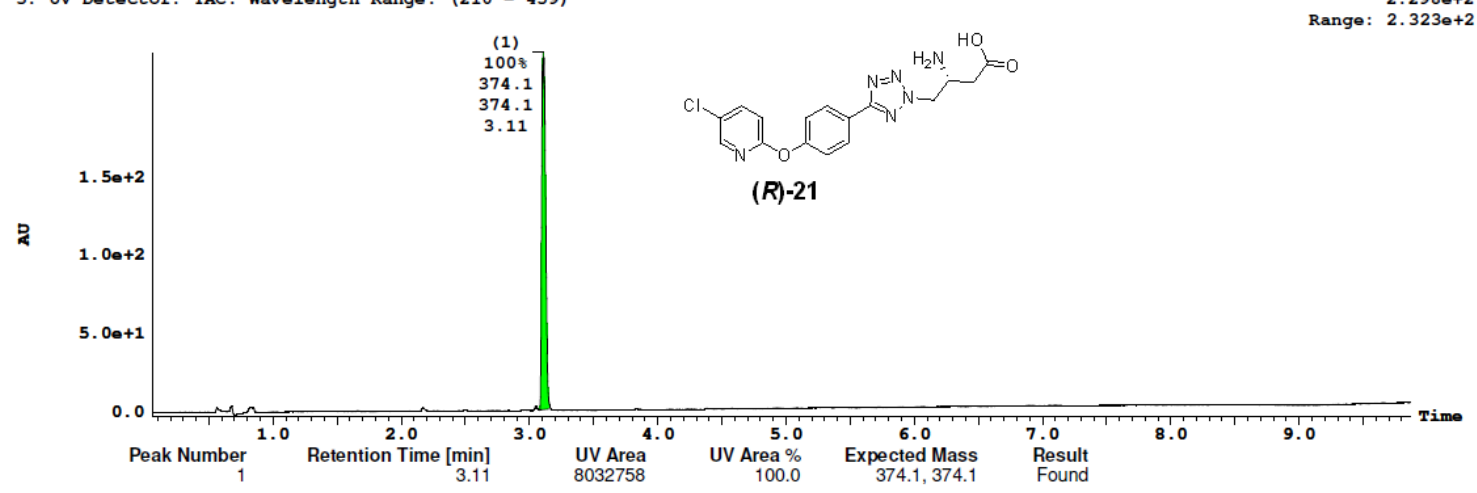


Instrument [BS_SQ16] WSJ-507.1.07

3: UV Detector: TAC: Wavelength Range: $(210-450)$

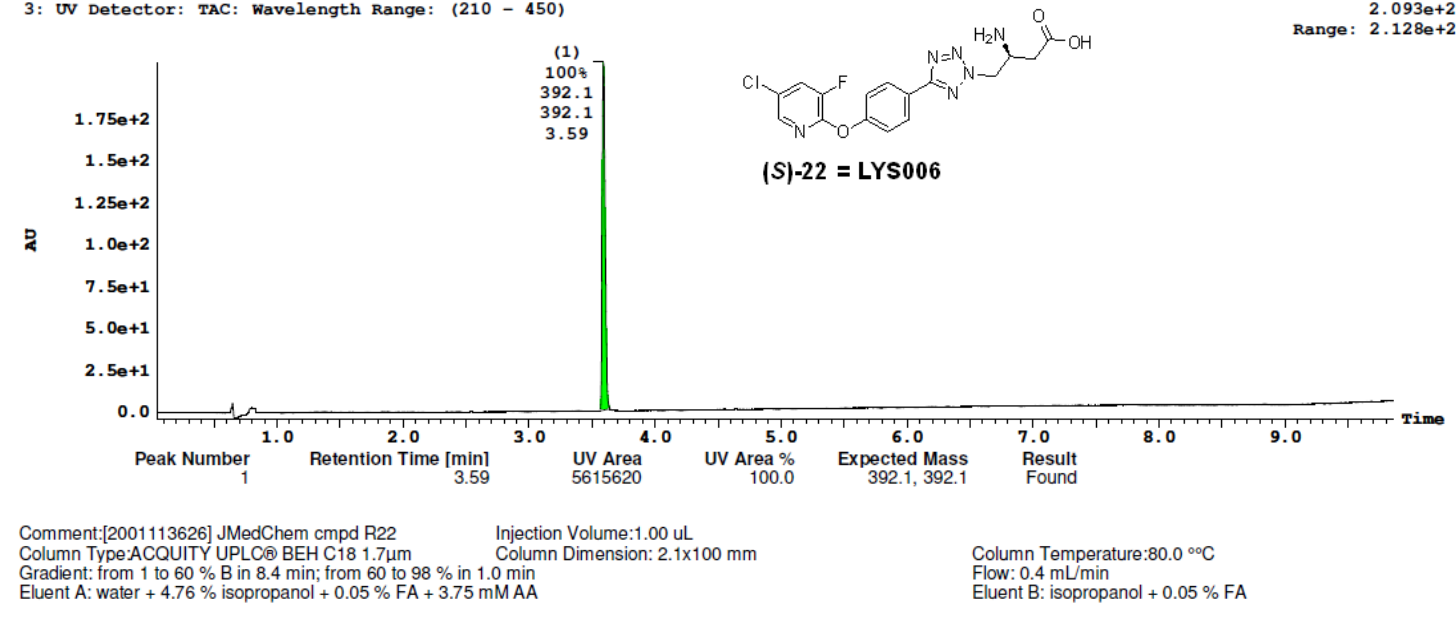

Instrument [BS_SQ09] WSJ-507.1.07

3: UV Detector: TAC: Wavelength Range: $(210-450)$

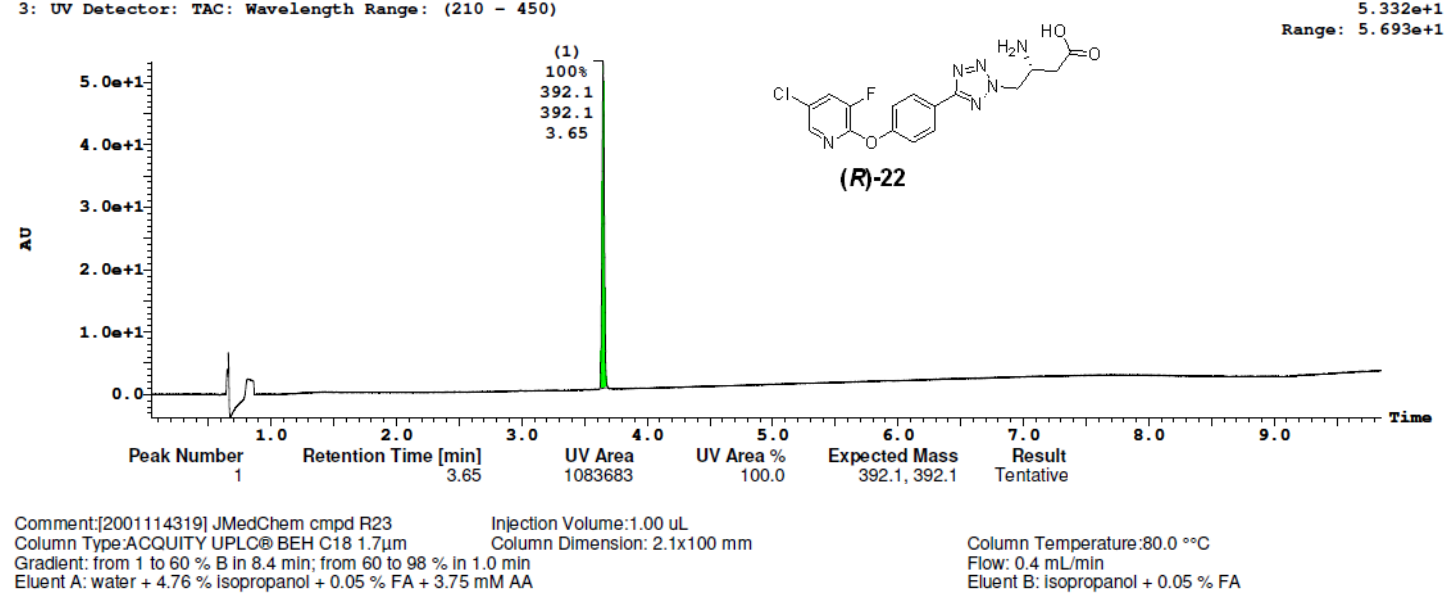

Instrument [BS_SQ09] WSJ-507.1.07

3: UV Detector: TAC: wavelength Range: $(210-450)$

$8.57 e+1$

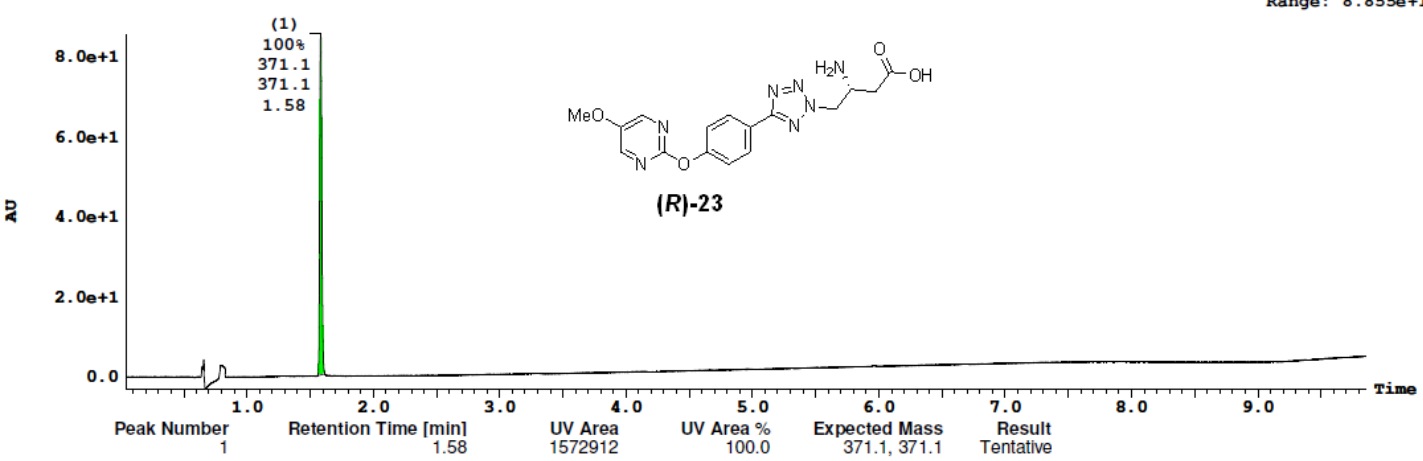


Table S1. Smiles and data for compounds 1-30.

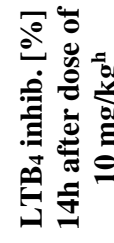

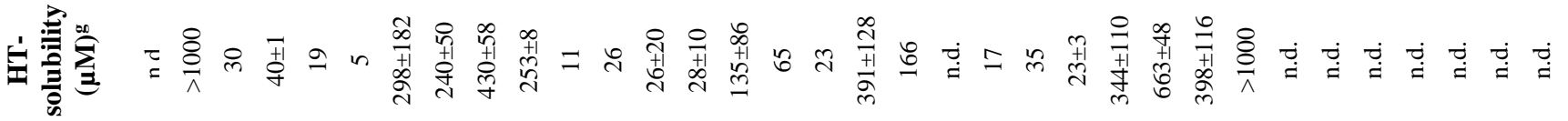

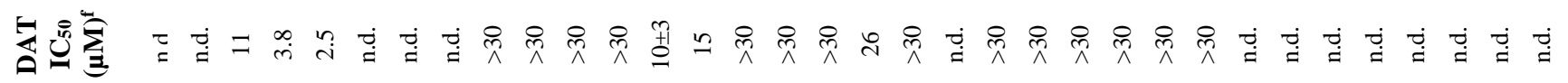

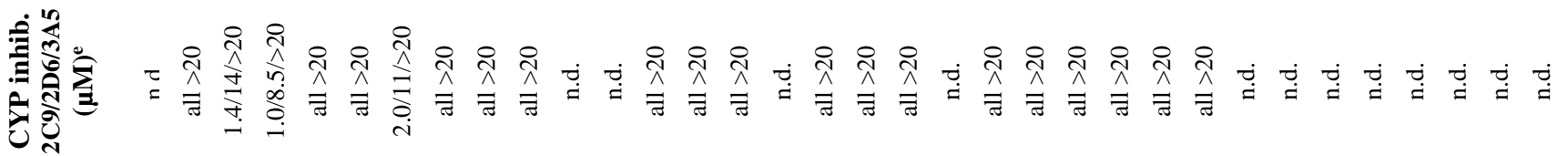

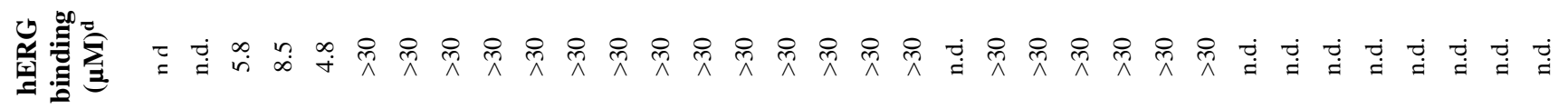

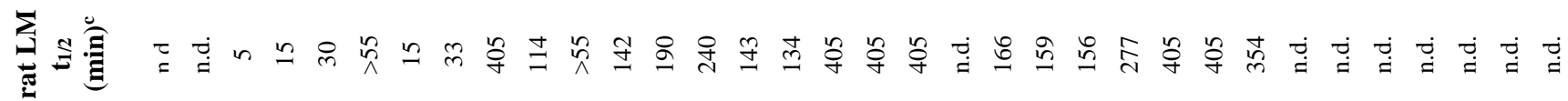

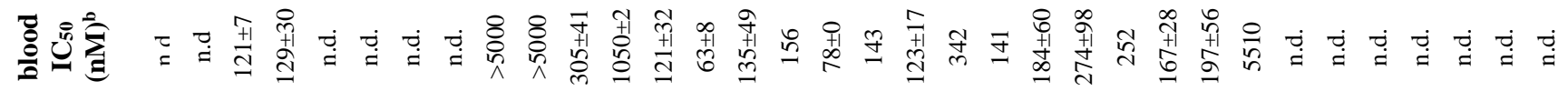

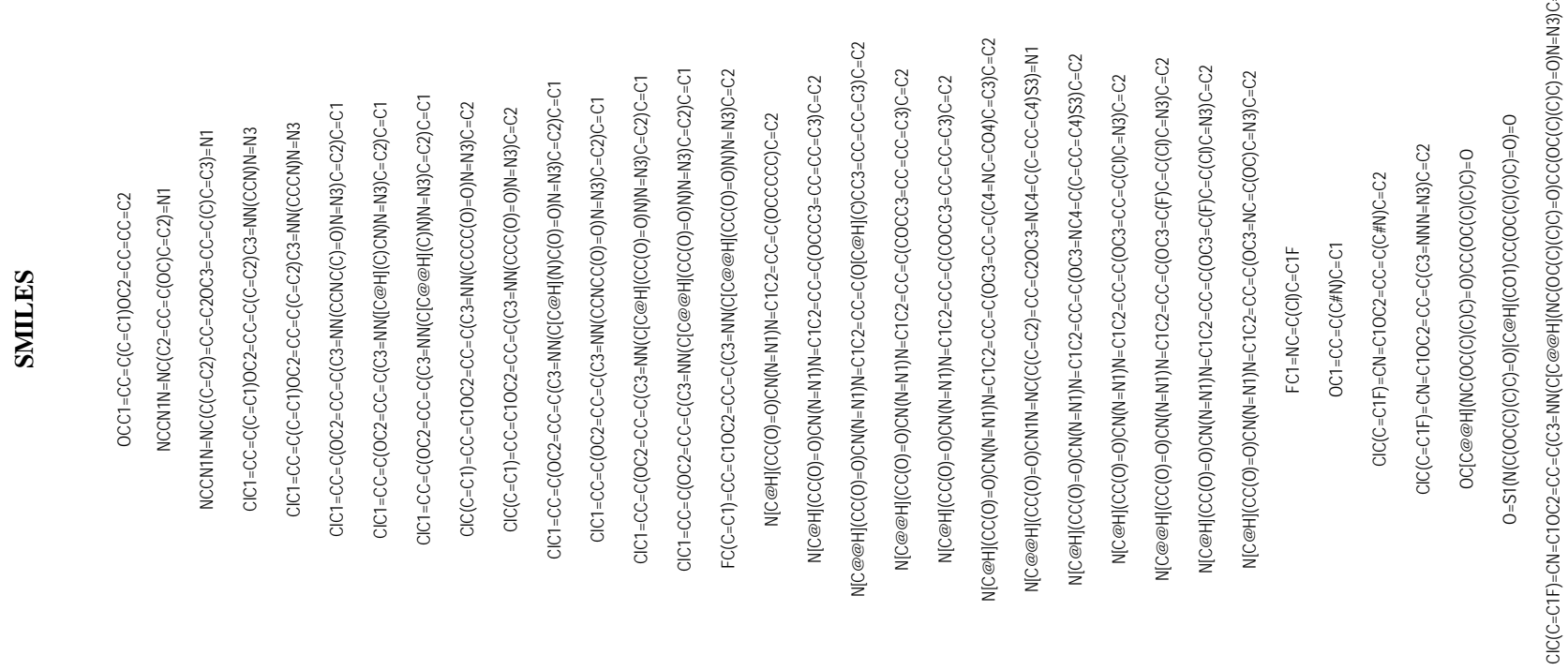

产 


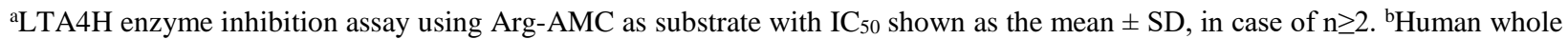
blood ionophore stimulated LTB4 release assay with $\mathrm{IC}_{50}$ shown as the mean $\pm \mathrm{SD}$, in case of $\mathrm{n} \geq 2$. ${ }^{\mathrm{C}}$ Metabolic stability in rat liver microsomes supplemented with NADPH. ${ }^{\mathrm{d} B i n d i n g}$ assay using radiolabeled ${ }^{3} \mathrm{H}$-dofetilide and HEK293 cell membranes expressing human recombinant HERG $\mathrm{K}^{+}$channels. ${ }^{4}$ Reversible Inhibition of CYP3A4 (midazolam), CYP2C9 (diclofenac) and CYP2D6 (bufuralol) in human liver microsomes. ${ }^{5}$ DAT radioligand binding assay on human recombinant DAT with IC50 shown as the mean of 1-6 assessments. ${ }^{6}$ EEquilibrated solubility in aqueous buffer at $\mathrm{pH}$ 6.8. ${ }^{7}$ hinhibition of $\mathrm{LTB}_{4}$ release from ex-vivo ionophorestimulated mouse blood $14 \mathrm{~h}$ after p.o. dose of compound compared to vehicle control given as the mean percent inhibition \pm SEM $(\mathrm{n} \geq 3)$.

Table S2. Inhibitory activity of LYS006 against selected Zn-containing enzymes.

\begin{tabular}{|c|c|c|c|c|}
\hline enzyme & $\mathrm{IC}_{50}(\mu \mathrm{M})$ & hill coefficient & $\begin{array}{l}\% \text { inhibition } \\
\text { @ highest conc. }\end{array}$ & highest conc. ( $\mu \mathrm{M})$ \\
\hline MMP01 & $>32$ & n.a. & -1.5 & $31.6^{\mathrm{a}}$ \\
\hline MMP01 & $>32$ & n.a. & -3.2 & $31.6^{\mathrm{a}}$ \\
\hline MMP02 & 51 & 4.0 & 94 & 100 \\
\hline MMP02 & $>32$ & n.a. & -3.9 & $31.6^{\mathrm{a}}$ \\
\hline МMP08 & $>32$ & n.a. & 0.012 & $31.6^{\mathrm{a}}$ \\
\hline MMP08 & $>32$ & n.a. & -1.1 & $31.6^{\mathrm{a}}$ \\
\hline MMP09 & $>32$ & n.a. & 3.1 & $31.6^{\mathrm{a}}$ \\
\hline MMP09 & $>32$ & n.a. & 8.6 & $31.6^{\mathrm{a}}$ \\
\hline MMP12 & $>32$ & n.a. & -4 & $31.6^{\mathrm{a}}$ \\
\hline MMP12 & $>32$ & n.a. & -3.9 & $31.6^{\mathrm{a}}$ \\
\hline MMP13 & $>32$ & n.a. & -4.1 & $31.6^{\mathrm{a}}$ \\
\hline MMP13 & $>32$ & n.a. & -4.6 & $31.6^{\mathrm{a}}$ \\
\hline MMP14 & $>32$ & n.a. & 25 & $31.6^{\mathrm{a}}$ \\
\hline MMP14 & $>32$ & n.a. & -4.4 & $31.6^{\mathrm{a}}$ \\
\hline TACE & 36 & 2.8 & 95 & 100 \\
\hline TACE & 43 & 1.8 & 101 & 100 \\
\hline
\end{tabular}

${ }^{\mathrm{a}}$ Compound interference $>31.6 \mu \mathrm{M}$. 


\section{STRUCTURAL BIOLOGY}

\section{Enzyme expression and purification}

Full-length LTA4H containing and N-terminal (His)6-tag followed by a (Gly3-Ser) 3 spacer and a human rhinovirus 3C (PreScission) cleavage site which leaves a Gly-Pro N-terminal overhang was expressed in E.coli BL21 (DE3) star cells. Cell lysate was applied to Nickle-NTA Super flow resin (Qiagen). The protein was eluted with a gradient of 20-500 mM imidazole, and subjected to fusion tag removal by precision site cleavage overnight. Finally, the LTA4H was further purified by size exclusion chromatography (Superdex SPX75/16/60; GE Health care). The resulting protein was estimated to be $>95 \%$ pure, homogeneous by SDS-PAGE, and reverse phase HPLC. N-terminal sequencing and Mass spectrometry (Q-Tof, Micromass) further confirmed the identity of the protein.

\section{Co-crystallization and X-ray structure determination}

Thin, plate-like crystals were obtained by the sitting drop vapor diffusion method at $17^{\circ} \mathrm{C}$ after mixing $0.2 \mu \mathrm{L}$ of LTA4H/ligand complex at $8 \mathrm{mg} / \mathrm{mL}$ protein concentration with an equal volume of reservoir solution consisting of $90.0 \mathrm{mM}$ Imidazol, $5.0 \mathrm{mM}$ Yattrium Chloride, $74.0 \mathrm{mM}$ Sodium accetae, pH6.5 in 96-well Cryschem plates. Crystals were harvested from their mother liquor with 20\% glycerol as cryo-protectant and directly flash-frozen in liquid nitrogen.

Data sets were collected at $1.000 \AA$ wavelength with a PILATUS 6M detector at the Swiss Light Source beamline X10SA (Villigen, Switzerland). Diffraction images recorded at $0.25^{\circ}$ oscillation angle wedges were processed and scaled using XDS and XSCALE respectively, in the APRV program suite. ${ }^{8}$ Data collection and processing statistics are summarized in table S2.

An initial structure was obtained by molecular replacement using Phaser $^{9}$ and the previously published coordinates of the protein component of the LTA4H structure (PDB entry 3FH5 ) as a search model. Manual rebuilding of the model and subsequent structure refinement were carried out in Coot $^{10}$ and auto Buster ${ }^{11}$ respectively. The ligand structure was built into unbiased Fo-Fc difference electron density calculated by auto Buster. Structural illustrations were prepared with PyMOL (www.pymol.org). Final structure refinement statistics are summarized in table S2. Refined coordinates are deposited in the PDB database (PDB IDs 7AUZ, 7AV0, 7AV1 and 7AV2). 


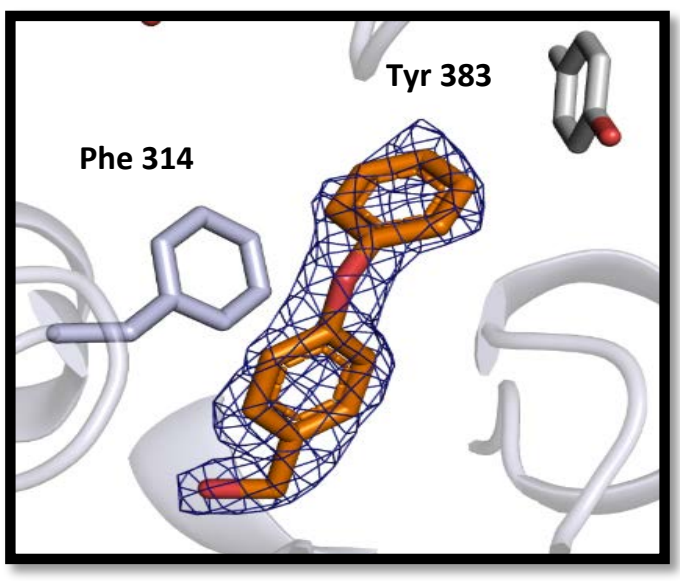

Fragment 1

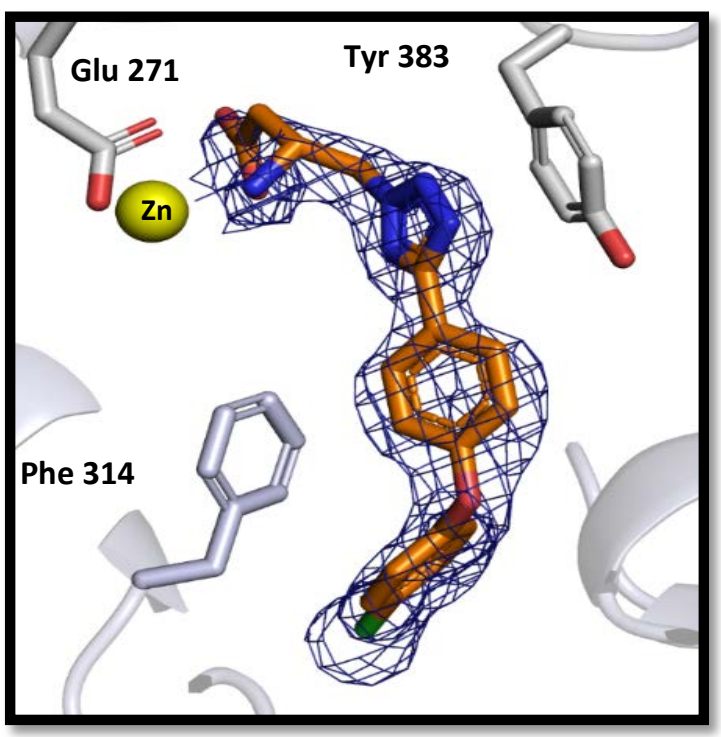

Compound $(R)-13$

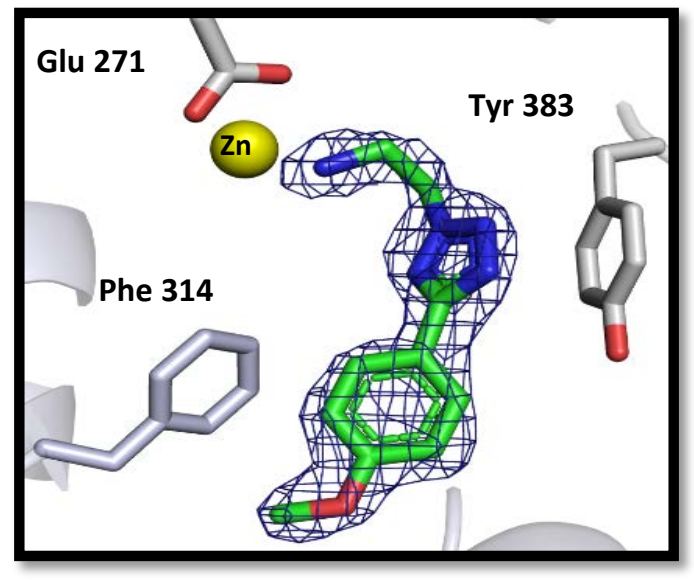

Fragment 2

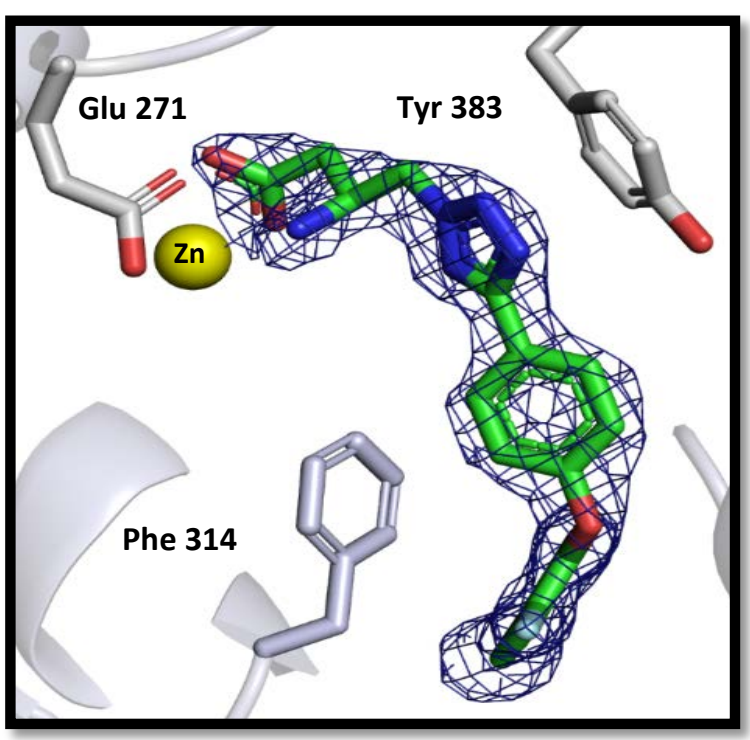

Compound (S)-22

Figure S1. Electron density (2Fo-Fc map) shown as blue mesh. 


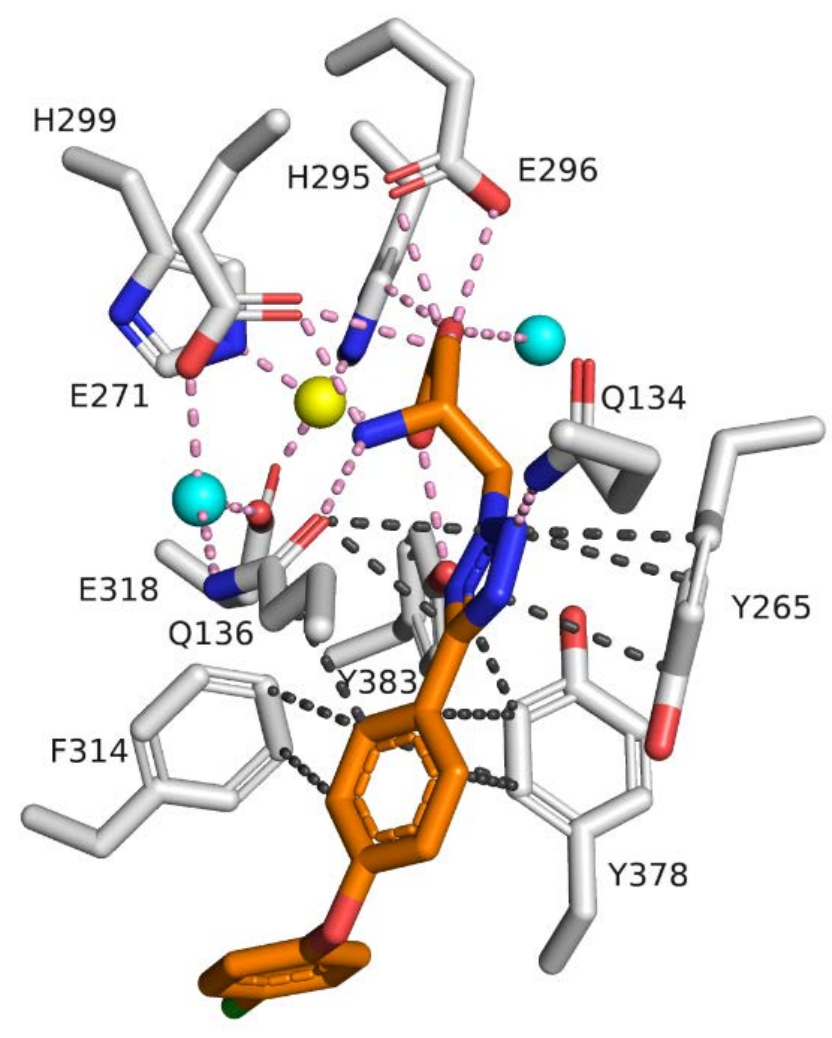

Compound $(R)-13$

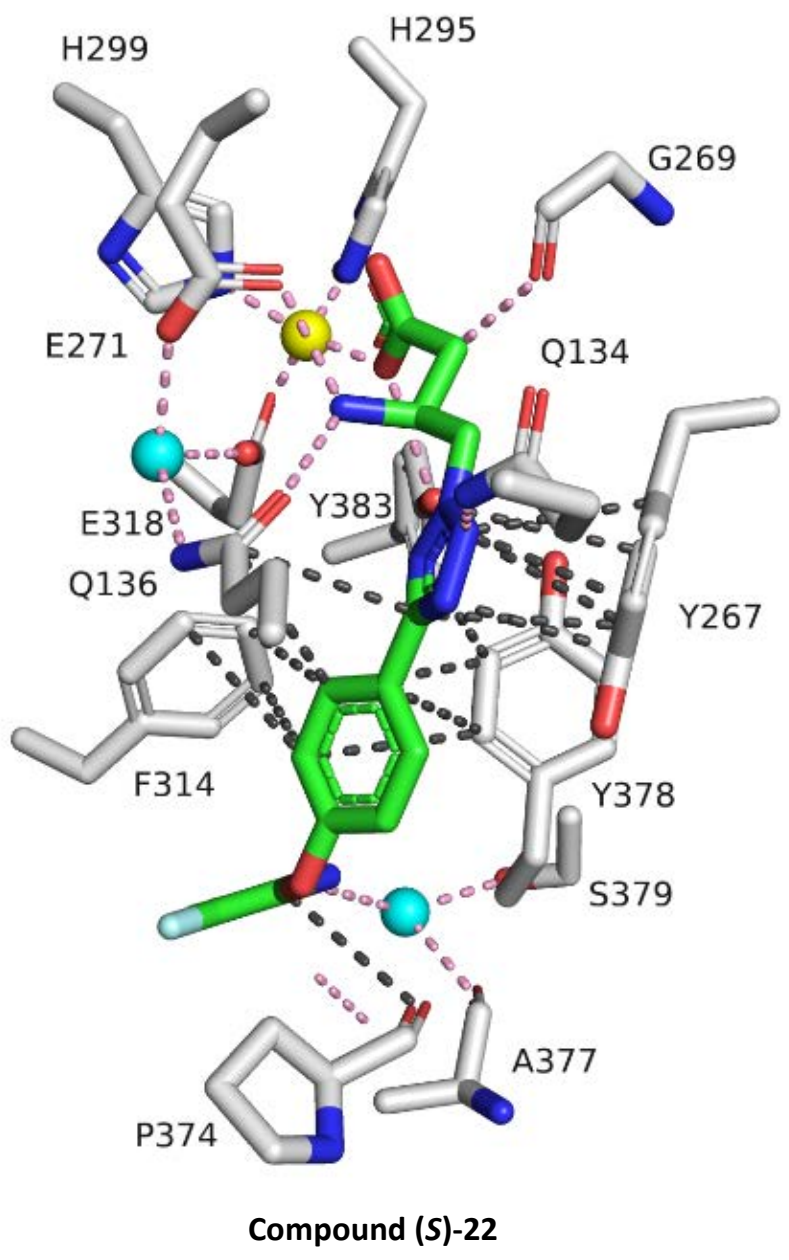

Figure S2. LTA4H-inhibitor interactions shown as dotted lines (hydrogen bonds in pink, pi-pi interactions in grey color). Waters and zinc are shown as cyan and yellow spheres, respectively. 
Table S3. Crystal data collection and refinement statistics

LTA4H-Fragment 1 LTA4H-Fragment 2 LTA4H-compound (S)-22 LTA4H-compound (R)-13

\begin{tabular}{|c|c|c|c|c|}
\hline \multicolumn{5}{|l|}{ A. Data collection } \\
\hline Space group & $P 2{ }_{1} 2_{1} 2_{1}$ & $P 2{ }_{1} 2_{1} 2_{1}$ & $P 2{ }_{1} 2_{1} 2_{1}$ & $P 2{ }_{1}{ }_{1} 2_{1}$ \\
\hline Unit-cell dimensions $(\AA)$ & $\begin{array}{l}a=77.8 b=87.0 c \\
=98.0\end{array}$ & $\begin{array}{l}a=77.8 b=87.1 c= \\
98.9\end{array}$ & $a=78.1 b=86.7 c=98.9$ & $a=78.2 b=87.1 c=99.6$ \\
\hline Resolution $(\AA)$ & 50-1.95 & 50-1.79 & 50-1.9 & 50-1.9 \\
\hline $\begin{array}{l}\text { Observations; unique } \\
\text { reflections }\end{array}$ & 272119,49278 & 352987,63418 & 355115,53693 & 357113,54201 \\
\hline Completeness(\%) & $99.3(99.4)^{\star}$ & $99.9(98.2)^{\star}$ & $99.9(100)^{\star}$ & $99.9(99.9)^{*}$ \\
\hline $1 / \sigma$ & $11.9(2.7)^{\star}$ & $14.2(2.60)^{*}$ & $10.5(1.8)^{\star}$ & $12.6(2.6)^{\star}$ \\
\hline$R_{\text {merge }}$ & $14.2(83.3)^{\star}$ & $9.6(74.8)^{*}$ & $14.8(90.5)^{\star}$ & $13.7(95.5)^{*}$ \\
\hline \multicolumn{5}{|l|}{ B. Refinement statistics } \\
\hline Resolution $(\AA)$ & 29-1.95 & 24-1.79 & $24-1.9$ & $24-1.9$ \\
\hline Reflections & 49278 & 63418 & 53639 & 54196 \\
\hline$R$-factor, $R_{\text {free }}$ & $16.3,20.8$ & $15.8,19.5$ & $17.2,20.6$ & $15.6,19.4$ \\
\hline Total number of protein atoms & 4852 & 4852 & 4852 & 4852 \\
\hline $\begin{array}{l}\text { Water atoms, and other } \\
\text { heteroatoms }\end{array}$ & 641,673 & 820,853 & 625,670 & 688,732 \\
\hline RMS bonds ( $\AA$ ), angles (deg.) & $0.01,1.03^{\circ}$ & $0.01,1.0^{\circ}$ & $0.01,1.00^{\circ}$ & $0.01,1.0^{\circ}$ \\
\hline
\end{tabular}

*Numbers in parentheses refer to the highest resolution shell 


\section{REFERENCES}

(1) Davies, D. R.; Mamat, B.; Magnusson, O. T.; Christensen, J.; Haraldsson, M. H.; Mishra, R.; Pease, B.; Hansen, E.; Singh, J.; Zembower, D.; Kim, H.; Kiselyov, A. S.; Burgin, A. B.; Gurney, M. E.; Stewart, L. J. Discovery of leukotriene A4 hydrolase inhibitors using metabolomics biased fragment crystallography. J. Med. Chem. 2009, 52, 4694-4715.

(2) Tanis, V. M.; Bacani, G. M.; Blevitt, J. M.; Chrovian, C. C.; Crawford, S.; De Leon, A.; Fourie, A. M.; Gomez, L.; Grice, C. A.; Herman, K.; Kearney, A. M.; Landry-Bayle, A. M.; LeeDutra, A.; Nelson, J.; Riley, J. P.; Santillán, A., Jr.; Wiener, J. J.; Xue, X.; Young, A. L. Azabenzthiazole inhibitors of leukotriene A4 hydrolase. Bioorg. Med. Chem. Lett. 2012, 22, 75047511.

(3) Diamine derivatives as inhibitors of leukotriene A4 hydrolase. US2007/155726, 2007.

(4) Finlayson, K.; Turnbull, L.; January, C. T.; Sharkey, J.; Kelly, J. S. [3H]Dofetilide binding to herg transfected membranes: a potential high throughput preclinical screen. Eur. J. Pharmacol. 2001, 430, 147-148.

(5) Zimmerlin, A.; Trunzer, M.; Faller, B. CYP3A time-dependent inhibition risk assessment validated with 400 reference drugs. Drug Metab. Dispos. 2011, 39, 1039-1046.

(6) Pristupa, Z. B.; Wilson, J. M.; Hoffman, B. J.; Kish, S. J.; Niznik, H. B. Pharmacological heterogeneity of the cloned and native human dopamine transporter: disassociation of [3H]WIN 35,428 and [3H]GBR 12,935 binding. Mol. Pharmacol. 1994, 45, 125-135.

(7) Zhou, L.; Yang, L.; Tilton, S.; Wang, J. Development of a high throughput equilibrium solubility assay using miniaturized shake-flask method in early drug discovery. J. Pharm. Sci. 2007, 96, 3052-3071.

(8) Kroemer, M.; Dreyer, M. K.; Wendt, K. U. APRV - a program for automated data processing, refinement and visualization. Acta Crystallogr., Sect. D: Biol. Crystallogr. 2004, 60, 1679-1682.

(9) McCoy, A. J.; Grosse-Kunstleve, R. W.; Adams, P. D.; Winn, M. D.; Storoni, L. C.; Read, R. J. Phaser crystallographic software. J. Appl. Crystallogr. 2007, 40, 658-674.

(10) Emsley, P.; Cowtan, K. Coot: Model-building tools for molecular graphics. Acta Crystallogr., Sect. D: Biol. Crystallogr. 2004, 60, 2126-2132.

(11) Bricogne, G.; Vonrhein, C.; Flensburg, C.; Schiltz, M.; Paciorek, W. Generation, representation and flow of phase information in structure determination: recent developments in and around SHARP 2.0. Acta Crystallogr., Sect. D: Biol. Crystallogr. 2003, 59, 2023-2030. 